\title{
Normalized ground states for semilinear elliptic systems with critical and subcritical nonlinearities*
}

\author{
Houwang $\mathbf{L i}^{1} \&$ Wenming Zou ${ }^{2}$ \\ 1. Department of Mathematical Sciences, Tsinghua University, Beijing 100084, China. \\ 2. Department of Mathematical Sciences, Tsinghua University, Beijing 100084, China.
}

\begin{abstract}
In the present paper, we study the normalized solutions with least energy to the following system:

$$
\begin{cases}-\Delta u+\lambda_{1} u=\mu_{1}|u|^{p-2} u+\beta r_{1}|u|^{r_{1}-2}|v|^{r_{2}} u & \text { in } \mathbb{R}^{N} \\ -\Delta v+\lambda_{2} v=\mu_{2}|v|^{q-2} v+\beta r_{2}|u|^{r_{1}}|v|^{r_{2}-2} v & \text { in } \mathbb{R}^{N} \\ \int_{\mathbb{R}^{N}} u^{2}=a_{1}^{2} \text { and } \int_{\mathbb{R}^{N}} v^{2}=a_{2}^{2}, & \end{cases}
$$

where $p, r_{1}+r_{2}<2^{*}$ and $q \leq 2^{*}$. To this purpose, we study the geometry of the Pohozaev manifold and the associated minimizition problem. Under some assumptions on $a_{1}, a_{2}$ and $\beta$, we obtain the existence of the positive normalized ground state solution to the above system.
\end{abstract}

Key words: Semilinear elliptic system; Normalized ground states; Pohozaev manifold; Sobolev critical.

2010 Mathematics Subject Classification: 35J50, 35J15, 35J60.

*This work is supported by NSFC(11801581,11025106, 11371212, 11271386); E-mails: li-hw17@mails.tsinghua.edu.cn \& zouwm@mail.tsinghua.edu.cn 


\section{Introduction}

We recall the following Schrödinger system:

$$
\left\{\begin{array}{l}
-i \frac{\partial}{\partial t} \Phi_{1}=\Delta \Phi_{1}+\mu_{1}\left|\Phi_{1}\right|^{p-2} \Phi_{1}+\beta r_{1}\left|\Phi_{1}\right|^{r_{1}-2}\left|\Phi_{2}\right|^{r_{2}} \Phi_{1}, \\
-i \frac{\partial}{\partial t} \Phi_{2}=\Delta \Phi_{2}+\mu_{2}\left|\Phi_{2}\right|^{q-2} \Phi_{1}+\beta r_{2}\left|\Phi_{1}\right|^{r_{1}}\left|\Phi_{2}\right|^{r_{2}-2} \Phi_{2}, \\
\Phi_{j}=\Phi_{j}(x, t) \in \mathbb{C},(x, t) \in \mathbb{R}^{N} \times \mathbb{R}, j=1,2,
\end{array}\right.
$$

where $i$ is the imaginary unit, $\mu_{1}, \mu_{2}$ and $\beta$ are constants, which comes from various physical phenomena, such as mean-field modles for binary mixtures of Bose-Einstein condensates, or binary gases of fermion atoms in degenerate quantum states (Bose-Fermi mixtures, Fermi-Fermi mixtures), see [1, 2, 8, 19] for more physical background. Physically, system 1.1 has the nature of conservation of mass, that is the following two norms

$$
\int_{\mathbb{R}^{N}}\left|\Phi_{1}(t, x)\right|^{2} \mathrm{~d} x \quad \text { and } \quad \int_{\mathbb{R}^{N}}\left|\Phi_{2}(t, x)\right|^{2} \mathrm{~d} x
$$

are independent of $t \in \mathbb{R}$. Moreover, the $L^{2}$-norms $\left|\Phi_{1}(t, \cdot)\right|_{2}$ and $\left|\Phi_{2}(t, \cdot)\right|_{2}$ have important physical significance, for example, in Bose-Einstein condensates, $\left|\Phi_{1}(t, \cdot)\right|_{2}$ and $\left|\Phi_{2}(t, \cdot)\right|_{2}$ represent the number of particles of each component; in nonlinear optics framwork, $\left|\Phi_{1}(t, \cdot)\right|_{2}$ and $\left|\Phi_{2}(t, \cdot)\right|_{2}$ represent the power supply. Therefore it is natural to consider the masses as preserved, and the solution of 1.1) with prescribed mass is called normalized solution.

In order to study the solitary wave solution of 1.1 , we set $\Phi_{1}(x, t)=e^{i \lambda_{1} t} u(x)$ and $\Phi_{2}(x, t)=e^{i \lambda_{2} t} v(x)$. Then the system 1.1 is reduced to the general elliptic system:

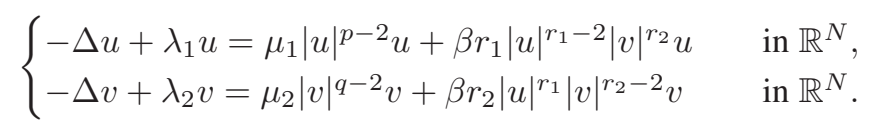

And the existence of normalized solutions to (1.2) can be formulated as follows: given $a_{1}, a_{2}>0$, we aim to find $(u, v) \in H^{1}\left(\mathbb{R}^{N}\right) \times H^{1}\left(\mathbb{R}^{N}\right)$ and $\left(\lambda_{1}, \lambda_{2}\right) \in \mathbb{R}^{2}$ such that

$$
\begin{cases}-\Delta u+\lambda_{1} u=\mu_{1}|u|^{p-2} u+\beta r_{1}|u|^{r_{1}-2}|v|^{r_{2}} u & \text { in } \mathbb{R}^{N} \\ -\Delta v+\lambda_{2} v=\mu_{2}|v|^{q-2} v+\beta r_{2}|u|^{r_{1}}|v|^{r_{2}-2} v & \text { in } \mathbb{R}^{N} \\ \int_{\mathbb{R}^{N}} u^{2}=a_{1}^{2} \quad \text { and } \quad \int_{\mathbb{R}^{N}} v^{2}=a_{2}^{2} . & \end{cases}
$$

Throughout the paper, we treat 1.3 in cases $\mu_{1}, \mu_{2}, \beta>0$, which is the so-called self-focusing and attractive interaction, and we require also

$$
N \geq 3, \quad r_{1}, r_{2}>1, \quad 2<p<2^{*}, \quad 2<r_{1}+r_{2}<2^{*}, \quad 2<q \leq 2^{*},
$$

where $2^{*}=\frac{2 N}{N-2}$ is the Sobolev critical exponent. These constants are prescribed while the parameters $\lambda_{1}, \lambda_{2}$ are unknown. It is easy to see that a normalized solution of 1.3 can be found as a critical point of the energy functional

$$
I(u, v)=\int_{\mathbb{R}^{N}} \frac{1}{2}\left(|\nabla u|^{2}+\left|\nabla v^{2}\right|\right)-\frac{1}{p} \mu_{1}|u|^{p}-\frac{1}{q} \mu_{2}|v|^{p}-\beta|u|^{r_{1}}|v|^{r_{2}}
$$

under the constraint $S_{a_{1}} \times S_{a_{2}}$, where

$$
S_{a}=\left\{u \in H^{1}\left(\mathbb{R}^{N}\right): \int_{\mathbb{R}^{N}} u^{2}=a^{2}\right\},
$$

and the parameters $\lambda_{1}, \lambda_{2}$ appear as Lagrangian multipliers. In this paper, we are particularly interested in the normalized ground states 
Definition 1.1. We say that $\left(u_{0}, v_{0}\right)$ is a normalized ground state of system (1.3), if it is a solution to (1.3) having minimal energy amoung all the normalized solutions:

$$
I\left(u_{0}, v_{0}\right)=\inf \left\{I(u, v):(u, v) \text { solves (1.3) for some }\left(\lambda_{1}, \lambda_{2}\right) \in \mathbb{R}^{2}\right\} \text {. }
$$

The search for normalized ground states of system $1.3 \mathrm{~b}$ is a challenging and interesting problem. The presence of the $L^{2}$-constraint makes the methods developed to deal with unconstraint problems unavailable, and new technical difficulties arise. One of the main difficulties is the lack of the compactness of the constraint Palais-Smale sequences. Indeed it is hard to check that the weak limits of the constraint Palais-Smale sequences lie in the constraint $S_{a_{1}} \times S_{a_{2}}$, since the embeddings $H^{1}\left(\mathbb{R}^{N}\right) \hookrightarrow L^{2}\left(\mathbb{R}^{N}\right)$ and even $H_{\text {rad }}^{1}\left(\mathbb{R}^{N}\right) \hookrightarrow L^{2}\left(\mathbb{R}^{N}\right)$ are not compact. Moreover, the $L^{2}$-constraint induces a new critical exponent, the $L^{2}$-critical exponent

$$
\bar{p}=2+\frac{4}{N} .
$$

This is the threshold exponent for the boundedness of the energy functional $I(u, v)$. If the problem is purely $L^{2}$-subcritical i.e., $2<p, q, r_{1}+r_{2}<\bar{p}$, then $I(u, v)$ is bounded from below on $S_{a_{1}} \times S_{a_{2}}$. In this case, T. Gou and L. Jeanjean [11] obtained the compactness of the minimizing sequence of $I(u, v)$ constrianed on $S_{a_{1}} \times S_{a_{2}}$, and proved the existence of a normalized ground state as a global minimizer. However, if one of $p, q, r_{1}+r_{2}$ is greater than $\bar{p}$, i.e., $L^{2}$-supercritical, then $I(u, v)$ is unbounded from below and from above on $S_{a_{1}} \times S_{a_{2}}$. In the cases $2<p, q<\bar{p}<r_{1}+r_{2}<2^{*}$ and $2<r_{1}+r_{2}<\bar{p}<p, q<2^{*}$, T. Gou and L. Jeanjean [12] proved the existence of a normalized ground state; in the cases $\bar{p}<p, q, r_{1}+r_{2}<2^{*}$, using the Pohozaev manifold and mountain pass lemma, T. Bartsch, L. Jeanjean and N. Soave (See [5, 4]) proved the existence of a normalized ground state for large $\beta$, below we will give more detailed comparisons on these results. For other conclusions about the existence and mulplicity of the normalized solutions for Schrödinger equations on the whole space, we refer to [3, 12, 5, 4, 6, 21, 22, 14].

We note that in [22], N. Soave considered the following nonlinear Schrödinger equation with combined power nonlinearities:

$$
-\Delta u=\lambda u+\mu|u|^{q-2} u+|u|^{2^{*}-2} u \quad \text { in } \mathbb{R}^{N}, N \geq 3
$$

with prescribed mass

$$
\int_{\mathbb{R}^{N}}|u|^{2}=a^{2}
$$

in the Sobolev critical case. For a $L^{2}$-subcritical, $L^{2}$-critical, and $L^{2}$-supercritical perturbation $\mu|u|^{q-2} u$, the author proved several existence/non-existence and stability/instability results. He obtained a constraint PalaisSmale sequence with an additional property by studying the geometry of the corrsponding Pohozaev manifold, and he proved the compactness of this special constraint Palais-Smale sequence under some energy level. We are motivated by [22] to study the system [1.3). However, we deal with a system, which is different from the scalar equation: the appearence of the coupled item makes the geometry of the Pohozaev manifold more complicated; the compactness of constraint Palais-Smale sequence is hard to get.

For simplicity, let $r=r_{1}+r_{2}$ and

$$
\gamma_{p}=\frac{N(p-2)}{2 p}\left\{\begin{aligned}
<\frac{2}{p}, & \text { if } 2<p<\bar{p}, \\
=\frac{2}{p}, & \text { if } p=\bar{p}, \quad \text { and } \quad \gamma_{2^{*}}=1 . \\
>\frac{2}{p}, & \text { if } \bar{p}<p<2^{*},
\end{aligned}\right.
$$


As in [21, 22], the following Pohozaev manifold will play a special role in the proof:

$$
\mathcal{P}_{a_{1}, a_{2}}=\left\{(u, v) \in S_{a_{1}} \times S_{a_{2}}: P(u, v)=0\right\},
$$

where

$$
P(u, v)=\int_{\mathbb{R}^{N}}|\nabla u|^{2}+|\nabla v|^{2}-\gamma_{p} \mu_{1}|u|^{p}-\gamma_{q} \mu_{2}|v|^{q}-r \gamma_{r} \beta|u|^{r_{1}}|v|^{r_{2}} .
$$

As a consequence of the Pohozaev identity, any solution of (1.3) belongs to $\mathcal{P}_{a_{1}, a_{2}}$. So if $(u, v) \in \mathcal{P}_{a_{1}, a_{2}}$ is a minimizer of the constraint minimization

$$
m\left(a_{1}, a_{2}\right)=\inf _{(u, v) \in \mathcal{P}_{a_{1}, a_{2}}} I(u, v),
$$

and $(u, v)$ solves system (1.2) for some $\lambda_{1}, \lambda_{2}$, then $(u, v)$ is a normalized ground state of (1.3). To study the minimization problem (1.10), we introduce a dilition operation preserving the $L^{2}$-norm: for $u \in S_{a}$ and $s \in \mathbb{R}$,

$$
s \star u(x):=e^{\frac{N s}{2}} u\left(e^{s} x\right) \text { for a.e. } x \in \mathbb{R}^{N} .
$$

Then $s \star u \in S_{a}$. Define $s \star(u, v)=(s \star u, s \star v)$ and the fiber maps

$$
\begin{aligned}
\Phi_{(u, v)}(s): & =I(s \star(u, v)) \\
& =\int_{\mathbb{R}^{N}} \frac{e^{2 s}}{2}\left(|\nabla u|^{2}+|\nabla v|^{2}\right)-\frac{e^{p \gamma_{p} s}}{p} \mu_{1}|u|^{p}-\frac{e^{q \gamma_{q} s}}{q} \mu_{2}|v|^{q}-e^{r \gamma_{r} s} \beta|u|^{r_{1}}|v|^{r_{2}} .
\end{aligned}
$$

By direct computation, we have $\Phi_{(u, v)}^{\prime}(s)=P(s \star(u, v))$ and

$$
\mathcal{P}_{a_{1}, a_{2}}=\left\{(u, v) \in S_{a_{1}} \times S_{a_{2}}: \Phi_{(u, v)}^{\prime}(0)=0\right\} .
$$

In this direction, we decompose $\mathcal{P}_{a_{1}, a_{2}}$ into disjoint unions $\mathcal{P}_{a_{1}, a_{2}}=\mathcal{P}_{a_{1}, a_{2}}^{+} \cup \mathcal{P}_{a_{1}, a_{2}}^{0} \cup \mathcal{P}_{a_{1}, a_{2}}^{-}$, where

$$
\begin{aligned}
& \mathcal{P}_{a_{1}, a_{2}}^{+}:=\left\{(u, v) \in S_{a_{1}} \times S_{a_{2}}: \Phi_{(u, v)}^{\prime \prime}(0)>0\right\}, \\
& \mathcal{P}_{a_{1}, a_{2}}^{0}:=\left\{(u, v) \in S_{a_{1}} \times S_{a_{2}}: \Phi_{(u, v)}^{\prime \prime}(0)=0\right\}, \\
& \mathcal{P}_{a_{1}, a_{2}}^{-}:=\left\{(u, v) \in S_{a_{1}} \times S_{a_{2}}: \Phi_{(u, v)}^{\prime \prime}(0)<0\right\} .
\end{aligned}
$$

We see that the monotonicity and convexity of $\Phi_{(u, v)}(s)$ will strongly affect the structure of $\mathcal{P}$ and hence have a strong impact on the minimization problem (1.10).

Now, we state our main results. As we have stated, throughout this paper, we require $\mu_{1}, \mu_{2}, \beta, a_{1}, a_{2}>0$ and $r_{1}, r_{2}>1$. For the convenience of description, let

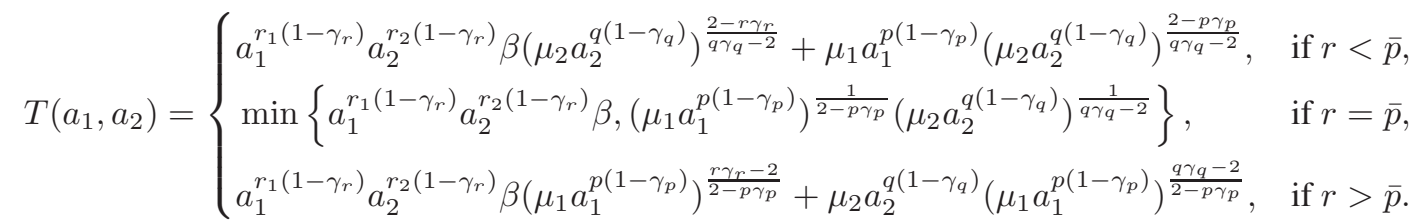

Then we have a result concerning a mixed situation. 
Theorem 1.1. Suppose $3 \leq N \leq 4,2<p<\bar{p}<q \leq 2^{*}, r<2^{*}, r_{2}<2$, then there exists a constant $\alpha_{0}=\alpha_{0}(p, q, r, N)>0$ such that if $T\left(a_{1}, a_{2}\right)<\alpha_{0}$, then (1.3) has a positive normalized ground state.

Remark 1.1. The assumption $r_{2}<2$ is used to control the energy level, and the assumption $T\left(a_{1}, a_{2}\right)<\alpha_{0}$ is applied to ensure that the Pohozaev manifold has a good geometry. We note that for fixed $\mu_{1}, \mu_{2}, \beta>0$, the constant $T\left(a_{1}, a_{2}\right)<\alpha_{0}$ holds as long as $a_{1} a_{2}$ small enough.

We also obtain a result about the normalized ground state for purely $L^{2}$-supercritical case.

Theorem 1.2. Suppose $3 \leq N \leq 4, \bar{p}<p, q, r<2^{*}$, then

(1) there exists a $\beta_{0}>0$ such that (1.3) has a positive normalized ground state for any $\beta>\beta_{0}$;

(2) if further $r_{1}, r_{2}<2$, then (1.3) has a positive normalized ground state for any $\beta>0$.

Remark 1.2. The first conclusion of Theorem [.2 is similar to the results in [4] 5], but the second one is a new result. Particularly, let $N=4, p=q=2 r_{1}=2 r_{2} \in\left(\bar{p}, 2^{*}\right)$, then according to the second conlusion of Theorem 1.2 the following system

$$
\begin{cases}-\Delta u+\lambda_{1} u=\mu_{1}|u|^{p-2} u+\beta r_{1}|u|^{\frac{p}{2}-2}|v|^{\frac{p}{2}} u & \text { in } \mathbb{R}^{4}, \\ -\Delta v+\lambda_{2} v=\mu_{2}|v|^{p-2} v+\beta r_{2}|u|^{\frac{p}{2}}|v|^{\frac{p}{2}-2} v & \text { in } \mathbb{R}^{4}, \\ \int_{\mathbb{R}^{4}} u^{2}=a_{1}^{2}, \quad \int_{\mathbb{R}^{4}} v^{2}=a_{2}^{2}, & \end{cases}
$$

has a positive normalized ground state for any $a_{1}, a_{2}, \beta>0$.

The paper is organized as follows. In Section 2 we collect some preliminary results which will be used from time to time in the paper. Theorems 1.1 1.2 are proved in Sections 4, 5 respectively. In Appendix B, we give a proof of a regularity result. Throughout the paper we use the notation $|u|_{p}$ to denote the $L^{p}\left(\mathbb{R}^{N}\right)$ norm, and we simply write $H^{1}=H^{1}\left(\mathbb{R}^{N}\right), H=H^{1}\left(\mathbb{R}^{N}\right) \times H^{1}\left(\mathbb{R}^{N}\right)$. Similarly, $H_{r}^{1}$ denotes the subspace of funtions in $H^{1}$ which are radial symmetric with respect to 0 , and $H_{r}=H_{r}^{1} \times H_{r}^{1}, S_{a, r}=S_{a} \cap H_{r}^{1}$. The symbol $\|\cdot\|$ denotes the norm in $H^{1}$ or $H$. Denoting by $u^{*}$ the symmetric decreasing rearrangement of $u \in H^{1}$, we recall that (see [18]) for $p, q>1$

$$
\left|\nabla u^{*}\right|_{2} \leq|\nabla u|_{2}, \quad\left|u^{*}\right|_{p}=|u|_{p} \quad \text { and } \quad \int_{\mathbb{R}^{N}}\left|u^{*}\right|^{p}\left|v^{*}\right|^{q} \geq \int_{\mathbb{R}^{N}}|u|^{p}|v|^{q}
$$

The capital letters $C_{1}, C_{2}, \cdots$ denote positive constants which may depend on $N, p, q, r_{1}, r_{2}$, whose precise values can change from line to line.

\section{Preliminaries}

In this section, we summarize several results which will be used in the rest disscussion.

For $N \geq 3,2<p \leq 2^{*}$, the Gagliardo-Nirenberg inequality is

$$
|u|_{p} \leq \mathcal{C}_{N, p}|\nabla u|_{2}^{\gamma_{p}}|u|_{2}^{1-\gamma_{p}}, \quad \forall u \in H^{1}
$$

where $\gamma_{p}$ is defined by (1.7). For a special case of 2.1], if $p=2^{*}$, then denoting $\mathcal{S}=\mathcal{C}_{N, 2^{*}}^{-2}$, we have the Sobolev inequality

$$
\mathcal{S}|u|_{2^{*}}^{2} \leq|\nabla u|_{2}^{2}, \quad \forall u \in D^{1,2}\left(\mathbb{R}^{N}\right)
$$


where $D^{1,2}\left(\mathbb{R}^{N}\right)$ is the completion of $C_{c}^{\infty}\left(\mathbb{R}^{N}\right)$ with respect to the norm $\|u\|_{D^{1,2}}:=|\nabla u|_{2}$. We observe that the functional $I(u, v)$ defined in (1.5) is well defined and is of class $C^{1}$. Throughout this paper, we denote

$$
\left\{\begin{array}{l}
\mathcal{D}_{1}=\left(\frac{\max \left\{r_{1}, r_{2}\right\}}{r}\right)^{\frac{r \gamma_{r}}{2}} \mathcal{C}_{N, r}^{r} a_{1}^{r_{1}\left(1-\gamma_{r}\right)} a_{2}^{r_{2}\left(1-\gamma_{r}\right)}, \\
\mathcal{D}_{2}=\frac{1}{p} \mu_{1} \mathcal{C}_{N, p}^{p} a_{1}^{p\left(1-\gamma_{p}\right)}, \\
\mathcal{D}_{3}=\frac{1}{q} \mu_{2} \mathcal{C}_{N, q}^{q} a_{2}^{q\left(1-\gamma_{q}\right)} .
\end{array}\right.
$$

Then we have

$$
\begin{aligned}
\int|u|^{r_{1}}|v|^{r_{2}} & \leq\left(\int\left|u_{1}\right|^{r}\right)^{\frac{r_{1}}{r}}\left(\int\left|u_{2}\right|^{r}\right)^{\frac{r_{2}}{r}} \\
& \leq \mathcal{C}_{N, r} a_{1}^{r_{1}\left(1-\gamma_{r}\right)} a_{2}^{r_{2}\left(1-\gamma_{r}\right)}\left(\int|\nabla u|^{2}\right)^{\frac{r_{1} \gamma_{r}}{2}}\left(\int|\nabla v|^{2}\right)^{\frac{r_{2} \gamma_{r}}{2}} \\
& \leq \mathcal{C}_{N, r} a_{1}^{r_{1}\left(1-\gamma_{r}\right)} a_{2}^{r_{2}\left(1-\gamma_{r}\right)}\left(\frac{r_{1}}{r} \int|\nabla u|^{2}+\frac{r_{2}}{r} \int|\nabla v|^{2}\right)^{\frac{r \gamma_{r}}{2}} \\
& \leq \mathcal{D}_{1}\left(\int|\nabla u|^{2}+|\nabla v|^{2}\right)^{\frac{r \gamma_{r}}{2}}
\end{aligned}
$$

Substituting 2.3)-2.4 into (1.5), we obtain

$$
\begin{aligned}
I(u, v) & \geq \frac{1}{2}\left(\int|\nabla u|^{2}+|\nabla v|^{2}\right)-\mathcal{D}_{1} \beta\left(\int|\nabla u|^{2}+|\nabla v|^{2}\right)^{\frac{r \gamma_{r}}{2}}-\mathcal{D}_{2}|\nabla u|_{2}^{p \gamma_{p}}-\mathcal{D}_{3}|\nabla v|_{2}^{q \gamma_{q}} \\
& \geq h\left(\left(\int|\nabla u|^{2}+|\nabla v|^{2}\right)^{\frac{1}{2}}\right),
\end{aligned}
$$

where $h(t):(0,+\infty) \rightarrow \mathbb{R}$ defined by

$$
h(t)=\frac{1}{2} t^{2}-\mathcal{D}_{1} \beta t^{r \gamma_{r}}-\mathcal{D}_{2} t^{p \gamma_{p}}-\mathcal{D}_{3} t^{q \gamma_{q}} .
$$

We now focus on the Sobolev subcritical and critical nonlinear Schrödinger equations with prescribed $L^{2}$-norm. For fixed $a>0, \mu>0,2<p \leq 2^{*}$, we search for $(u, \lambda) \in H^{1} \times \mathbb{R}$ solving

$$
\left\{\begin{array}{l}
-\Delta u+\lambda u=\mu|u|^{p-2} u \quad \text { in } \mathbb{R}^{N}, \\
\int_{\mathbb{R}^{N}} u^{2}=a^{2}, \quad u \in H^{1} .
\end{array}\right.
$$

Solutions of (2.7) can be found as the critical points of $E_{p, \mu}: H^{1} \rightarrow \mathbb{R}$

$$
E_{p, \mu}(u)=\int_{\mathbb{R}^{N}} \frac{1}{2}|\nabla u|^{2}-\frac{1}{p} \mu|u|^{p},
$$

constrained on $S_{a}$, and the parameter $\lambda$ appears as Lagrangian multiplier. Back to the problem (2.7), testing the equation with $u$, we get

$$
\int_{\mathbb{R}^{N}}|\nabla u|^{2}+\lambda \int_{\mathbb{R}^{N}} u^{2}=\mu \int_{\mathbb{R}^{N}}|u|^{p},
$$


and then combined with the Pohozaev identity

$$
\frac{N-2}{2} \int_{\mathbb{R}^{N}}|\nabla u|^{2}+\frac{N}{2} \lambda \int_{\mathbb{R}^{N}} u^{2}=\frac{N}{p} \mu \int_{\mathbb{R}^{N}}|u|^{p},
$$

it follows that

$$
\lambda \int_{\mathbb{R}^{N}} u^{2}=\left(1-\gamma_{p}\right) \mu \int_{\mathbb{R}^{N}}|u|^{p}
$$

Hence, if $p<2^{*}$, then we must have $\lambda>0$. Therefore, by scaling, the equation (2.7) is equivalent to

$$
-\Delta w+w=|w|^{p-2} w \quad \text { in } \mathbb{R}^{N}, \quad w \in H^{1} .
$$

While if $p=2^{*}$, we have $\lambda=0$, then by scaling, the equation (2.7) is equivalent to

$$
-\Delta w=|w|^{2^{*}-2} w \quad \text { in } \mathbb{R}^{N}, \quad w \in D^{1,2}\left(\mathbb{R}^{N}\right) .
$$

Since the positive solutions of (2.9) (2.10) are studied clearly, the existence of normalized solutions of (2.7) can be obtained by scaling. However, there are still some special properties that need to be clarified. To be precise, we introduce the Pohozaev manifold for single equations

$$
\mathcal{T}_{a, p, \mu}:=\left\{u \in S_{a}: \int_{\mathbb{R}^{N}}|\nabla u|^{2}-\gamma_{p} \mu|u|^{p}=0\right\},
$$

and the constraint minimizition problem

$$
m_{p}^{\mu}(a)=\inf _{u \in \mathcal{T}_{a, p, \mu}} E_{p, \mu}(u)
$$

It is easy to see that

$$
m\left(a_{1}, 0\right)=m_{p}^{\mu_{1}}\left(a_{1}\right) \quad \text { and } \quad m\left(0, a_{2}\right)=m_{q}^{\mu_{2}}\left(a_{2}\right) .
$$

We have the following lemmas.

Lemma 2.1. Suppose $N \geq 3, \mu, a>0$ and $2<p<2^{*}, p \neq \bar{p}$, then up to a translation, (2.7) has a unique positive solution $u_{p, \mu} \in \mathcal{T}_{a, p, \mu}$ with $\lambda>0$. Moreover,

(1) if $p<\bar{p}$, then

$$
m_{p}^{\mu}(a)=\inf _{u \in S_{a}} E_{p, \mu}(u)=E_{p, \mu}\left(u_{p, \mu}\right)<0
$$

(2) if $p>\bar{p}$, then

$$
m_{p}^{\mu}(a)=\inf _{u \in S_{a}} \max _{t \in \mathbb{R}} E_{p, \mu}(t \star u)=\max _{t \in \mathbb{R}} E_{p, \mu}\left(t \star u_{p, \mu}\right)=E_{p, \mu}\left(u_{p, \mu}\right)>0 ;
$$

Finally, for both cases $m_{p}^{\mu}(a)$ is strictly decreasing with respect to $a>0$.

Proof. By [16, 10], up to a translation, $w_{p, \mu}$ is the unique positive solution of [2.9], which is radial symmetric and decreasing with respect to 0 . Since $p \neq \bar{p}$, by scaling we obtain the unique solution of 2.7

$$
u_{p, \mu}=\left(\frac{\lambda}{\mu}\right)^{\frac{1}{p-2}} w_{p, \mu}\left(\lambda^{\frac{1}{2}} x\right) \quad \text { with } \quad \lambda=\left(\frac{a^{2}}{\left|w_{p, \mu}\right|_{2}^{2}} \mu^{\frac{2}{p-2}}\right)^{\frac{p-2}{2-p \gamma_{p}}} .
$$


Using the Pohozaev identity, it is easy to check that $u_{p, \mu} \in \mathcal{T}_{a, p, \mu}$. On the other hand, the equality of (2.1) is achieved by $w_{p, \mu}$, that is

$$
\left|w_{p, \mu}\right|_{p}=\mathcal{C}_{p, N}\left|\nabla w_{p, \mu}\right|_{2}^{\gamma_{p}}\left|w_{p, \mu}\right|_{2}^{1-\gamma_{p}},
$$

and taking a similar process as the one to get equation $(2.8)$, we obtain

$$
\left|\nabla w_{p, \mu}\right|_{2}^{2}=\gamma_{p}\left|w_{p, \mu}\right|_{p}^{p}
$$

Then combining (2.15) and (2.16), there is $\left|\nabla w_{p, \mu}\right|_{2}^{2-p \gamma_{p}}=\gamma_{p} C_{p, N}\left|w_{p, \mu}\right|_{2}^{p-p \gamma_{p}}$. So

$$
\begin{aligned}
E_{p, \mu}\left(u_{p, \mu}\right) & =\left(\frac{1}{2}-\frac{1}{p \gamma_{p}}\right) \int_{\mathbb{R}^{N}}\left|\nabla u_{p, \mu}\right|^{2} \\
& =\left(\frac{1}{2}-\frac{1}{p \gamma_{p}}\right) \mu^{-\frac{2}{p-2}} \lambda^{\frac{p-p \gamma_{p}}{p-2}}\left|\nabla w_{p, N}\right|_{2}^{2} \\
& =\left(\frac{1}{2}-\frac{1}{p \gamma_{p}}\right)\left(\mu a^{p-p \gamma_{p}}\right)^{\frac{2}{2-p \gamma_{p}}} \frac{\left|\nabla w_{p, N}\right|_{2}^{2}}{\left|w_{p, N}\right|_{2}^{\frac{2\left(p-p \gamma_{p}\right)}{2-p \gamma_{p}}}} \\
& =\left(\frac{1}{2}-\frac{1}{p \gamma_{p}}\right)\left(\gamma_{p} \mathcal{C}_{N, p} \mu a^{p-p \gamma_{p}}\right)^{\frac{2}{2-p \gamma_{p}}},
\end{aligned}
$$

which is negative if $p<\bar{p}$ and is positive if $p>\bar{p}$. To prove futher properties, let

$$
\begin{aligned}
\Phi_{u}(s) & :=E_{p, \mu}(s \star u) \\
& =\frac{e^{2 s}}{2} \int_{\mathbb{R}^{N}}|\nabla u|^{2}-\frac{e^{p \gamma_{p} s}}{p} \gamma_{p} \int_{\mathbb{R}^{N}} \mu|u|^{p} .
\end{aligned}
$$

For the case $p<\bar{p}$, we take $u \in S_{a}$, then a direct computation tells us that there exists a unique global minimizer $s_{u}$ for $\Phi_{u}(s)$ and $s_{u} \star u \in \mathcal{T}_{a, p, \mu}$. So

$$
E_{p, \mu}(u) \geq E_{p, \mu}\left(s_{u} \star u\right) \geq m_{p}^{\mu}(a) \geq \inf _{u \in S_{a}} E_{p, \mu}(u),
$$

which implies $m_{p}^{\mu}(a)=\inf _{u \in S_{a}} E_{p, \mu}(u)<0$. Taking a minimizing sequence $u_{n}$ for $\inf _{u \in S_{a}} E_{p, \mu}(u)$, we assume $u_{n} \geq 0, u_{n} \in H_{r}^{1}$ by insteading $u_{n}$ of $\left|u_{n}\right|^{*}$. The coerciveness of $\left.E_{p, \mu}\right|_{S_{a}}$ means that $u_{n}$ is bounded. Then up to a subsequence $u_{n} \rightarrow u_{0}$ in $H^{1}\left(\mathbb{R}^{N}\right), u_{n} \rightarrow u_{0}$ in $L^{p}\left(\mathbb{R}^{N}\right)$ and $u_{n} \rightarrow u_{0}$ a.e. in $\mathbb{R}^{N}$. So $u_{0} \geq 0$. We will prove that $u_{0}$ is a nontivial minimizer of $m_{p}^{\mu}(a)$. If $u_{0}=0$, then we have

$$
m_{p}^{\mu}(a)=\lim _{n \rightarrow \infty} E_{p, \mu}\left(u_{n}\right)=\lim _{n \rightarrow \infty} \frac{1}{2} \int_{\mathbb{R}^{N}}\left|\nabla u_{n}\right|^{2} \geq 0,
$$

in contradiction with $m_{p}^{\mu}(a)<0$. Hence, $0<\left|u_{0}\right|_{2} \leq a$. Suppose $\left|u_{0}\right|_{2} \neq a$, then

$$
m_{p}^{\mu}(a)=E_{p, \mu}\left(u_{n}\right)+o(1) \geq E_{p, \mu}\left(u_{0}\right) \geq m_{p}^{\mu}\left(\left|u_{0}\right|_{2}\right) .
$$

On the other hand, following the proof of [11, Lemma 3.1], we have

$$
m_{p}^{\mu}(a) \leq m_{p}^{\mu}\left(\left|u_{0}\right|_{2}\right)+m_{p}^{\mu}\left(a-\left|u_{0}\right|_{2}\right)<m_{p}^{\mu}\left(\left|u_{0}\right|_{2}\right),
$$

which is a contradiction. Thus $\left|u_{0}\right|_{2}=a, E_{p, \mu}\left(u_{0}\right)=m_{p}^{\mu}(a)$ and $u_{0}$ is a positive solution of 2.7). Then the uniqueness result implies $u_{0}=u_{p, \mu}$ and hence $m_{p}^{\mu}(a)=E_{p, \mu}\left(u_{p, \mu}\right)$. Recall 2.17), we know that $m_{p}^{\mu}(a)$ is strictly decreasing with respect to $a>0$.

Suppose now $p>\bar{p}$, then from [15, Lemma 2.10], there is $m_{p}^{\mu}(a)=\max _{t \in \mathbb{R}} E_{p, \mu}\left(t \star u_{p, \mu}\right)=E_{p, \mu}\left(u_{p, \mu}\right)$, and we can prove $m_{p}^{\mu}(a)=\inf _{u \in S_{a}} \max _{t \in \mathbb{R}} E_{p, \mu}(t \star u)$ similarly as[22, Proposition 2.2]. 
For the Sobolev critical case $p=2^{*}$, there is also a clear characterization about the positive solutions of (2.7) and the minimizition problem 2.12).

Lemma 2.2. Suppose $N \geq 3, \mu, a>0$ and $p=2^{*}$, then

$$
m_{2^{*}}^{\mu}(a)=\inf _{u \in S_{a}} \max _{t \in \mathbb{R}} E_{2^{*}, \mu}(t \star u)=\frac{1}{N} \mu^{-\frac{N-2}{2}} \mathcal{S}^{\frac{N}{2}}>0 .
$$

Moreover,

(1) if $N=3,4$, then 2.7 has no posotive solution for any $\lambda \in \mathbb{R}$, and in particular $m_{2^{*}}^{\mu}(a)$ is not achieved;

(2) if $N \geq 5$, then up to a translation, 2.7] has a unique positive solution $u_{2^{*}, \mu} \in \mathcal{T}_{a, 2^{*}, \mu}$ with $\lambda=0$, and

$$
m_{2^{*}}^{\mu}(a)=E_{2^{*}, \mu}\left(u_{2^{*}, \mu}\right) .
$$

Proof. For detailed proof, we refer to [22, Propesition 2.2].

In the following paper, we need the following result.

Lemma 2.3. Suppose $(u, v) \in H$ is a nonnegative solution of (1.2) with $2<p, q, r \leq 2^{*}$, then

(1) if $N=3,4$, then $u>0$ implies $\lambda_{1}>0$; $v>0$ implies $\lambda_{2}>0$;

(2) if $N \geq 5$, then $u>0$ implies $\lambda_{1} \geq 0$; $v>0$ implies $\lambda_{2} \geq 0$.

Proof. From Corollary B.1, we know that $(u, v)$ is a smooth solution. Suppose $u>0$ but $\lambda_{1}<0$, then

$$
-\Delta u=\left|\lambda_{1}\right| u+\mu_{1} u^{p-1}+\beta r_{1} u^{r_{1}-1} v^{r_{2}} \geq \min \left\{\left|\lambda_{1}\right|, \mu_{1}\right\} u^{\sigma} \quad \text { in } \mathbb{R}^{N},
$$

for any $1<\sigma<p-1$. Using a Liouville type theorem [20, Theorem 8.4], we deduce $u=0$, which is impossible since $u>0$. So $\lambda_{1} \geq 0$. Morevoer, if $N=3,4$ and $\lambda_{1}=0$, i.e.,

$$
-\Delta u=\mu_{1} u^{p-1}+\beta r_{1} u^{r_{1}-1} v^{r_{2}} \geq 0 \quad \text { in } \mathbb{R}^{N},
$$

then [13, Lemma A.2] implies that $u=0$, which is also a contradiction. So $\lambda_{1}>0$ when $N=3,4$.

Finally we recall a Brezis-Lieb type lemma.

Lemma 2.4. Suppose $\left(u_{n}, v_{n}\right) \subset H$ is a bounded sequence, $\left(u_{n}, v_{n}\right) \rightarrow(u, v)$ a.e. in $\mathbb{R}^{N}$ and $2 \leq r \leq$ $2^{*}, r_{1}, r_{2}>1$, then

$$
\lim _{n \rightarrow \infty} \int_{\mathbb{R}^{N}}\left|u_{n}\right|^{r_{1}}\left|v_{n}\right|^{r_{2}}-|u|^{r_{1}}|v|^{r_{2}}-\left|u_{n}-u\right|^{r_{1}}\left|v_{n}-v\right|^{r_{2}}=0
$$

\section{The mixed exponent case}

In this section, we assume $2<p<\bar{p}<q \leq 2^{*}, 2<r<2^{*}, r_{1}, r_{2}>1$. Recall the definition of $h(t)$ in (2.6), we have

Lemma 3.1. There exists a constant $\alpha_{1}>0$ such that if $T\left(a_{1}, a_{2}\right)<\alpha_{1}$, then the function $h(t)$ has exactly two critical points, one is a local minimum at negitive level, the other one is a global maximum at positive level. Futher, there exists $0<R_{0}<R_{1}$ such that $h\left(R_{0}\right)=h\left(R_{1}\right)=0$, and $h(t)>0$ iff $t \in\left(R_{0}, R_{1}\right)$. 
Remark 3.1. Let $l(t)=a t^{2}-b t^{r \gamma_{r}}-c t^{p \gamma_{p}}-d t^{q \gamma_{q}}$ with $2<p<\bar{p}<q \leq 2^{*}, 2<r<2^{*}$ and $a, b, c, d>0$. Then $l(t)$ has at most two critical points in $(0, \infty)$.

The proof of Lemma3.1 and Remark 3.1 is elementary but technique, we postpone it to the Appendix .

Lemma 3.2. There exists a constant $\alpha_{2}>0$ such that if $T\left(a_{1}, a_{2}\right)<\alpha_{2}$, then $\mathcal{P}_{a_{1}, a_{2}}^{0}=\emptyset$, and $\mathcal{P}_{a_{1}, a_{2}}$ is a $C^{1}$ submanifold in $H$ with codimension 3.

Proof. We first prove that $\mathcal{P}_{a_{1}, a_{2}}^{0}=\emptyset$ implies that $\mathcal{P}_{a_{1}, a_{2}}$ is a $C^{1}$ submanifold in $H$ with codimension 3. As we can see, $\mathcal{P}_{a_{1}, a_{2}}$ is defined by $P(u, v)=0, G(u)=0, F(v)=0$, where

$$
G(u)=a_{1}^{2}-\int_{\mathbb{R}^{N}} u^{2}, \quad F(v)=a_{2}^{2}-\int_{\mathbb{R}^{N}} v^{2} .
$$

It is sufficient to prove

$$
d(P, G, F): H \rightarrow \mathbb{R}^{3} \quad \text { is a surjective. }
$$

Suppose it is not true, by the independence of $d G(u)$ and $d F(v)$, there must be that $d P(u, v)$ is a linear combination of $d G(u)$ and $d F(v)$, i.e., there exist $\nu_{1}, \nu_{2} \in \mathbb{R}$ such that $(u, v)$ is a weak solution of

$$
\left\{\begin{array}{l}
-\Delta u+\nu_{1} u=\frac{p \gamma_{p}}{2} \mu_{1}|u|^{p-2} u+\frac{r \gamma_{r}}{2} \beta r_{1}|u|^{r_{1}-2}|v|^{r_{2}} u \quad \text { in } \mathbb{R}^{N}, \\
-\Delta v+\nu_{2} v=\frac{q \gamma_{q}}{2} \mu_{2}|v|^{q-2} v+\frac{r \gamma_{r}}{2} \beta r_{2}|u|^{r_{1}}|v|^{r_{2}-2} v \quad \text { in } \mathbb{R}^{N}, \\
|u|_{2}=a_{1}, \quad|v|_{2}=a_{2} .
\end{array}\right.
$$

Testing system 3.1 with $(u, v)$ and combining with the Pohozaev identity, we can conclude that

$$
2 \int_{\mathbb{R}^{N}}|\nabla u|^{2}+|\nabla v|^{2}=p \gamma_{p}^{2} \int_{\mathbb{R}^{N}} \mu_{1}|u|^{p}+q \gamma_{q}^{2} \int_{\mathbb{R}^{N}} \mu_{2}|v|^{q}+\left(r \gamma_{r}\right)^{2} \int_{\mathbb{R}^{N}} \beta|u|^{r_{1}}|v|^{r_{2}},
$$

which implies that $(u, v) \in \mathcal{P}_{a_{1}, a_{2}}^{0}$, a contradiction.

Now we prove that there exists a constant $\alpha_{2}>0$ such that $\mathcal{P}_{a_{1}, a_{2}}^{0}=\emptyset$ as long as $T\left(a_{1}, a_{2}\right)<\alpha_{2}$. Suppose there is a $(u, v) \in \mathcal{P}_{a_{1}, a_{2}}^{0}$. Let $\rho=\left(|u|_{2}^{2}+|v|_{2}^{2}\right)^{\frac{1}{2}}$ and

$$
\begin{aligned}
W(t):= & t \Phi_{(u, v)}^{\prime}(0)-\Phi_{(u, v)}^{\prime \prime}(0) \\
= & (t-2) \int_{\mathbb{R}^{N}}|\nabla u|^{2}+|\nabla v|^{2}-\left(t-p \gamma_{p}\right) \gamma_{p} \int_{\mathbb{R}^{N}} \mu_{1}|u|^{p} \\
& -\left(t-q \gamma_{q}\right) \gamma_{q} \int_{\mathbb{R}^{N}} \mu_{2}|v|^{q}-\left(t-r \gamma_{r}\right) r \gamma_{r} \int_{\mathbb{R}^{N}} \beta|u|^{r_{1}}|v|^{r_{2}} \\
= & 0 .
\end{aligned}
$$

We divide the proof into four different situations.

Case 1: $p \leq r<\bar{p}$. There is $p \gamma_{p} \leq r \gamma_{r}<2<q \gamma_{q}$. On the one hand, $W\left(r \gamma_{r}\right)=0$ implies

$$
\left(2-r \gamma_{r}\right) \rho^{2} \leq\left(q \gamma_{q}-r \gamma_{r}\right) \int_{\mathbb{R}^{N}} \mu_{2}|v|^{q} \leq\left(q \gamma_{q}-r \gamma_{r}\right) q \mathcal{D}_{3} \rho^{q \gamma_{q}} .
$$

It follows that $\rho \geq\left(\frac{q \gamma_{q}-r \gamma_{r}}{2-r \gamma_{r}} \frac{1}{q \mathcal{D}_{3}}\right)^{\frac{1}{q \gamma_{q}-2}}$. On the other hand, by $W\left(q \gamma_{q}\right)=0$, we obtain

$$
\begin{aligned}
\left(q \gamma_{q}-2\right) & =\left(q \gamma_{q}-p \gamma_{p}\right) \gamma_{p} \rho^{-2} \int_{\mathbb{R}^{N}} \mu_{1}|u|^{p}+\left(q \gamma_{q}-r \gamma_{r}\right) r \gamma_{r} \rho^{-2} \int_{\mathbb{R}^{N}} \beta|u|^{r_{1}}|v|^{r_{2}} \\
& \leq\left(q \gamma_{q}-p \gamma_{p}\right) \gamma_{p} p \mathcal{D}_{2} \rho^{p \gamma_{p}-2}+\left(q \gamma_{q}-r \gamma_{r}\right) r \gamma_{r} \mathcal{D}_{1} \beta \rho^{r \gamma_{r}-2} \\
& \leq C(p, q, r)\left(\mathcal{D}_{2} \mathcal{D}_{3}^{\frac{2-p \gamma_{p}}{q \gamma_{q}-2}}+\mathcal{D}_{1} \beta \mathcal{D}_{3}^{\frac{2-r \gamma_{r}}{q \gamma_{q}-2}}\right) .
\end{aligned}
$$


Therefore by the definitions of $\mathcal{D}_{1}, \mathcal{D}_{2}, \mathcal{D}_{3}$, we can choose an $\alpha_{2}>0$ such that

$$
\mathcal{D}_{2} \mathcal{D}_{3}^{\frac{2-p \gamma_{p}}{q \gamma_{q}-2}}+\mathcal{D}_{1} \beta \mathcal{D}_{3}^{\frac{2-r \gamma_{r}}{q \gamma_{q}-2}}<C(p, q, r)^{-1}\left(q \gamma_{q}-2\right)
$$

as long as $T\left(a_{1}, a_{2}\right)<\alpha_{2}$, then we get a contradiction. That is, $\mathcal{P}_{a_{1}, a_{2}}^{0}=\emptyset$ provided that $T\left(a_{1}, a_{2}\right)<\alpha_{2}$.

Case 2: $r<p<\bar{p}$. If we exchange the roles played by $\mathcal{D}_{2} t^{p \gamma_{p}}$ and $\mathcal{D}_{1} t^{r \gamma_{r}}$ in Case 1, then we can get a constant $\alpha_{2}$ with the required properties.

Case 3: $r=\bar{p}$. We first suppose $\alpha_{2}<\frac{1}{4}$, so that $\frac{1}{2}-\mathcal{D}_{1} \beta \in\left(\frac{1}{4}, \frac{1}{2}\right)$ when $\mathcal{D}_{1} \beta<\alpha_{2}$. Then completely analogous as Case 1, by combining $W\left(q \gamma_{q}\right)=0$ and $W\left(p \gamma_{p}\right)=0$, we can obtain the constant $\alpha_{2}$ with the required properties.

Case 4: $r>\bar{p}$. If $r \leq q$, then there is $p \gamma_{p}<2<r \gamma_{r} \leq q \gamma_{q}$ and again proceeding as Case 1, by combining $W\left(r \gamma_{r}\right)=0$ and $W\left(p \gamma_{p}\right)=0$, we can obtain the constant $\alpha_{2}$ with the required properties. If $r>q$, then there is $p \gamma_{p}<2<q \gamma_{q}<r \gamma_{r}$ and again proceeding as Case 1, by combining $W\left(q \gamma_{q}\right)=0$ and $W\left(p \gamma_{p}\right)=0$, we can obtain the constant $\alpha_{2}$ with the required properties.

Using Lemmas 3.1, 3.2 we can discribe the geometry of $\mathcal{P}_{a_{1}, a_{2}}$.

Lemma 3.3. If $T\left(a_{1}, a_{2}\right)<\min \left\{\alpha_{1}, \alpha_{2}\right\}$, then for every $(u, v) \in S_{a_{1}} \times S_{a_{2}}$, the function $\Phi_{(u, v)}(t)$ has exactly two critical points $s_{(u, v)}<t_{(u, v)}$ and two zero-points $c_{(u, v)}<d_{(u, v)}$ with $s_{(u, v)}<c_{(u, v)}<t_{(u, v)}<d_{(u, v)}$. Moreover:

(1) $s \star(u, v) \in \mathcal{P}_{a_{1}, a_{2}}^{+}$iff $s=s_{(u, v)} ; s \star(u, v) \in \mathcal{P}_{a_{1}, a_{2}}^{-}$iff $s=t_{(u, v)}$;

(2) $s_{(u, v)}<\log \frac{R_{0}}{\left(\int_{\mathbb{R} N}|\nabla u|^{2}+|\nabla v|^{2}\right)^{1 / 2}}$ and

$$
\Phi_{(u, v)}\left(s_{(u, v)}\right)=\inf \left\{\Phi_{(u, v)}(s): s \in\left(-\infty, \log \frac{R_{0}}{\left(\int_{\mathbb{R}^{N}}|\nabla u|^{2}+|\nabla v|^{2}\right)^{1 / 2}}\right)\right\} ;
$$

(3) $I\left(t_{(u, v)} \star(u, v)\right)=\max _{s \in \mathbb{R}} I(s \star(u, v))>0$;

(4) the maps $(u, v) \mapsto t_{(u, v)}$ and $(u, v) \mapsto s_{(u, v)}$ are of class $C^{1}$.

Proof. Let $(u, v) \in S_{a_{1}} \times S_{a_{2}}$. By (2.5), we have

$$
\Phi_{(u, v)}(s)=I(s \star(u, v)) \geq h\left(e^{s}\left(\int_{\mathbb{R}^{N}}|\nabla u|^{2}+|\nabla v|^{2}\right)^{1 / 2}\right),
$$

and then

$$
\Phi_{(u, v)}(s)>0, \quad \forall s \in\left(\log \frac{R_{0}}{\left(\int_{\mathbb{R}^{N}}|\nabla u|^{2}+|\nabla v|^{2}\right)^{1 / 2}}, \log \frac{R_{1}}{\left(\int_{\mathbb{R}^{N}}|\nabla u|^{2}+|\nabla v|^{2}\right)^{1 / 2}}\right) .
$$

Recalling the facts that $\Phi_{(u, v)}(-\infty)=0^{-}$and $\Phi_{(u, v)}(+\infty)=-\infty$, we see that $\Phi_{(u, v)}$ has at least two critical points $s_{(u, v)}<t_{(u, v)}$, where $s_{(u, v)}$ is local minimum point on $\left(-\infty, \log \frac{R_{0}}{\left(\int_{\mathbb{R}^{N}}|\nabla u|^{2}+|\nabla v|^{2}\right)^{1 / 2}}\right)$ at negetive level, and $t_{(u, v)}$ ia a global maximum point at positive level. On the other hand, from Remark $3, \Phi_{(u, v)}(s)$ has at most two critical points in $(-\infty,+\infty)$, which means that $\Phi_{(u, v)}(s)$ has exactly two critical points $s_{(u, v)}$ and $t_{(u, v)}$. Since $\Phi_{(u, v)}^{\prime}(s)=P(s \star(u, v))$, we know that $s \star(u, v) \in \mathcal{P}_{a_{1}, a_{2}}$ implies $s=s_{(u, v)}$ or $t_{(u, v)}$. Moreover, noticing $\Phi_{(u, v)}^{\prime \prime}\left(s_{(u, v)}\right) \geq 0, \Phi_{(u, v)}^{\prime \prime}\left(t_{(u, v)}\right) \leq 0$ and $\mathcal{P}_{a_{1}, a_{2}}^{0}=\emptyset$, we deduce that $s_{(u, v)} \star(u, v) \in \mathcal{P}_{a_{1}, a_{2}}^{+}$and $t_{(u, v)} \star(u, v) \in \mathcal{P}_{a_{1}, a_{2}}^{-}$. 
By the monotonicity, $\Phi_{(u, v)}$ has exactly two zero-points $c_{(u, v)}$ and $d_{(u, v)}$, with $s_{(u, v)}<c_{(u, v)}<t_{(u, v)}<$ $d_{(u, v)}$. It remains to show that the maps $(u, v) \mapsto t_{(u, v)}$ and $(u, v) \mapsto s_{(u, v)}$ are of class $C^{1}$. We apply the implicit function theorem on $\Psi(s, u, v)=\Phi_{(u, v)}^{\prime}(s)$. Using the fact that

$$
\begin{aligned}
& \Psi\left(s_{(u, v)}, u, v\right)=\Psi\left(t_{(u, v)} \star(u, v)\right)=0, \\
& \partial_{s} \Psi\left(s_{(u, v)}, u, v\right)=\Phi_{(u, v)}^{\prime \prime}\left(s_{(u, v)}\right)>0, \\
& \partial_{s} \Psi\left(s_{(u, v)}, u, v\right)=\Phi_{(u, v)}^{\prime \prime}\left(t_{(u, v)}\right)<0,
\end{aligned}
$$

and $\mathcal{P}_{a_{1}, a_{2}}^{0}=\emptyset$, there is $(u, v) \mapsto t_{(u, v)}$ and $(u, v) \mapsto s_{(u, v)}$ are of class $C^{1}$.

For $k>0$, let

$$
A_{R}:=\left\{(u, v) \in S_{a_{1}} \times S_{a_{2}}:\left(\int_{\mathbb{R}^{N}}|\nabla u|^{2}+|\nabla v|^{2}\right)^{1 / 2}<R\right\} .
$$

We have the following crucial estimates.

Lemma 3.4. Let $N \geq 3$ and $T\left(a_{1}, a_{2}\right)<\min \left\{\alpha_{1}, \alpha_{2}\right\}$. If $r_{2}<2$, then

$$
m\left(a_{1}, a_{2}\right)=\inf _{(u, v) \in A_{R_{0}}} I(u, v)<\min \left\{m\left(a_{1}, 0\right), m\left(0, a_{2}\right)\right\} .
$$

Proof. From Lemma 3.3, we have

$$
\mathcal{P}_{a_{1}, a_{2}}^{+}=\left\{s_{(u, v)} \star(u, v):(u, v) \in S_{a_{1}} \times S_{a_{2}}\right\} \subset A_{R_{0}},
$$

and

$$
m\left(a_{1}, a_{2}\right)=\inf _{\mathcal{P}_{a_{1}, a_{2}}} I(u, v)=\inf _{\mathcal{P}_{a_{1}, a_{2}}^{+}} I(u, v)<0 .
$$

Obviously $m\left(a_{1}, a_{2}\right) \geq \inf _{A_{R_{0}}} I(u, v)$. On the other hand, for any $(u, v) \in A_{R_{0}}$, since $0<\log \frac{R_{0}}{\left(\int_{\mathbb{R}^{N}}|\nabla u|^{2}+|\nabla v|^{2}\right)^{1 / 2}}$, there is

$$
m\left(a_{1}, a_{2}\right) \leq I\left(s_{(u, v)} \star(u, v)\right) \leq I(u, v) .
$$

It follows that $m\left(a_{1}, a_{2}\right)=\inf _{(u, v) \in A_{R_{0}}} I(u, v)$. Noting that $p<\bar{p}<q$ means $m\left(a_{1}, 0\right)<0<m\left(0, a_{2}\right)$, we only need to prove $m\left(a_{1}, a_{2}\right)<m\left(a_{1}, 0\right)$.

We now choose a proper test function to prove $m\left(a_{1}, a_{2}\right)<m\left(a_{1}, 0\right)$. From $h\left(R_{0}\right)=0$, we have $\frac{1}{2} R_{0}^{2}>$ $\mathcal{D}_{2} R_{0}^{p \gamma_{p}}$, that is $R_{0}^{2-p \gamma_{p}}>2 \mathcal{D}_{2}$. Let $\left(u, \lambda_{0}\right) \in S_{a_{1}} \times \mathbb{R}^{+}$be the unique function in Lemma2.1 with parameters $p, \mu_{1}, a_{1}$. It follows that

$$
|\nabla u|_{2}^{2}=\gamma_{p} \mu_{1}|u|_{p}^{p} \leq p \gamma_{p} \mathcal{D}_{2}|\nabla u|_{2}^{p \gamma_{p}}<R_{0}^{2-p \gamma_{p}}|\nabla u|_{2}^{p \gamma_{p}},
$$

which means $|\nabla u|_{2}<R_{0}$. Take $m>1$ such that $\frac{N}{2}-\frac{2}{r_{2}}<m<\frac{N}{2}-1$ and let

$$
\varphi(x) \in C_{0}^{\infty}\left(B_{2}(0)\right), \quad 0 \leq \varphi(x) \leq 1, \quad \varphi(x)=1 \text { in } B_{1}(0) .
$$

We define $v(x)=c \frac{\varphi(x)}{|x|^{m}} \in H^{1}$ with constant $c>0$ such that $v \in S_{a_{2}}$. Therefore, $(u, s \star v) \in A_{R_{0}}$ for $s \ll-1$. Let

$$
\alpha(s)=\int_{\mathbb{R}^{N}}|u|^{r_{1}}|s \star v|^{r_{2}}=C_{0} e^{\left(\frac{N}{2}-m\right) r_{2} s} \int_{\mathbb{R}^{N}} u^{r_{1}}(x) \frac{\varphi^{r_{2}}\left(e^{s} x\right)}{|x|^{m r_{2}}} .
$$


From the decay properties of [17], we get by replacing a variable that $u$ decays exponentially

$$
u(x)=O\left(|x|^{-\frac{N-2}{2}} e^{-\lambda_{0}^{\frac{1}{2}}|x|}\right), \quad \text { as }|x| \rightarrow \infty,
$$

and $|u(x)| \leq M$ in $\mathbb{R}^{N}$. Then

$$
0<\int_{\mathbb{R}^{N}} \frac{u^{r_{1}}(x)}{|x|^{m r_{2}}} \leq C\left(\int_{B_{R}(0)} \frac{1}{|x|^{m r_{2}}}+\int_{B_{R}(0)^{c}}|x|^{-\frac{(N-2) r_{1}}{2}-m r_{2}} e^{-\lambda_{0}^{\frac{1}{2}} r_{1}|x|}\right)<\infty .
$$

Thus by the Dominated Convergence Theorem, we obtain

$$
\lim _{s \rightarrow-\infty} \int_{\mathbb{R}^{N}} u^{r_{1}}(x) \frac{\varphi^{r_{2}}\left(e^{s} x\right)}{|x|^{m r_{2}}}=\int_{\mathbb{R}^{N}} u^{r_{1}}(x) \frac{1}{|x|^{m r_{2}}}=: C_{1} \in(0, \infty) .
$$

Hence $\alpha(s)=C_{0} e^{\theta s}\left(C_{1}+o(1)\right)>\frac{C_{0} C_{1}}{2} e^{\theta s}$ as $s \rightarrow-\infty$ where $\theta=\left(\frac{N}{2}-m\right) r_{2} \in(1,2)$. Finally we see that for some $s \ll-1$, there holds

$$
\begin{aligned}
m\left(a_{1}, a_{2}\right) & \leq I(u, s \star v) \\
& =E_{p, a_{1}, \mu}(u)+\frac{e^{2 s}}{2}|\nabla v|_{2}^{2}-\frac{e^{q \gamma_{q} s}}{q}|v|_{q}^{q}-\beta \alpha(s) \\
& <E_{p, a_{1}, \mu}(u)+\frac{e^{2 s}}{2}|\nabla v|_{2}^{2}-\frac{e^{q \gamma_{q} s}}{q}|v|_{q}^{q}-\beta \frac{C_{0} C_{1}}{2} e^{\theta s} \\
& <E_{p, a_{1}, \mu}(u)=m\left(a_{1}, 0\right) .
\end{aligned}
$$

Now we prove the compactness of Palais-Smale sequences.

Lemma 3.5. Suppose $N=3,4$ and $\mathcal{D}_{1} \beta<\frac{1}{4}$ when $r=\bar{p}$. Let $\left(u_{n}, v_{n}\right) \subset S_{a_{1}} \times S_{a_{2}}$ be a radial Palais-Smale sequence for $\left.I\right|_{S_{a_{1}} \times S_{a_{2}}}$ at level $m\left(a_{1}, a_{2}\right)$ with additional properties $P\left(u_{n}, v_{n}\right) \rightarrow 0$ and $u_{n}^{-}, v_{n}^{-} \rightarrow 0$ a.e. in $\mathbb{R}^{N}$. If

$$
m\left(a_{1}, a_{2}\right)<\min \left\{m\left(a_{1}, 0\right), m\left(0, a_{2}\right)\right\},
$$

then up to a subsequence, $\left(u_{n}, v_{n}\right) \rightarrow(u, v)$ in $H$, where $(u, v)$ is a positive solution of (1.2) for some $\lambda_{1}, \lambda_{2}>$ 0 .

Proof. We first prove that $\left(u_{n}, v_{n}\right)$ is bounded. Let $\rho_{n}=\left(\left|u_{n}\right|_{2}^{2}+\left|v_{n}\right|_{2}^{2}\right)^{\frac{1}{2}}$ and

$$
\begin{aligned}
Z_{n}(t):= & t I\left(u_{n}, v_{n}\right)-P\left(u_{n}, v_{n}\right) \\
= & \frac{t-2}{2} \int\left|\nabla u_{n}\right|^{2}+\left|\nabla v_{n}\right|^{2}-\frac{t-p \gamma_{p}}{p} \int \mu_{1}\left|u_{n}\right|^{p} \\
& -\frac{t-q \gamma_{q}}{q} \int \mu_{2}\left|v_{n}\right|^{q}-\left(t-r \gamma_{r}\right) \int \beta\left|u_{n}\right|^{r_{1}}\left|v_{n}\right|^{r_{2}} \\
\leq & C(t), \quad \forall n \geq 1 .
\end{aligned}
$$

We still disscuss it in four different situations.

Case 1: $r<\bar{p}$. From $Z_{n}\left(q \gamma_{q}\right) \leq C$, we get

$$
\frac{q \gamma_{q}-2}{2} \rho_{n}^{2} \leq C+\frac{q \gamma_{q}-p \gamma_{p}}{p} \int \mu_{1}\left|u_{n}\right|^{p}+\left(q \gamma_{q}-r \gamma_{r}\right) \int \beta\left|u_{n}\right|^{r_{1}}\left|v_{n}\right|^{r_{2}} \leq C\left(1+\rho_{n}^{p \gamma_{p}}+\rho_{n}^{r \gamma_{r}}\right),
$$

which implies that $\left(u_{n}, v_{n}\right)$ is bounded. 
Case 2: $r=\bar{p}$. Note that $r \gamma_{r}=2$. From $Z_{n}\left(q \gamma_{q}\right) \leq C$, we get

$$
\begin{aligned}
\frac{q \gamma_{q}-2}{2}\left(1-2 \mathcal{D}_{1} \beta\right) \rho_{n}^{2} & \leq C+\frac{q \gamma_{q}-2}{2} \rho_{n}^{2}-\left(q \gamma_{q}-2\right) \int \beta\left|u_{n}\right|^{r_{1}}\left|v_{n}\right|^{r_{2}} \\
& \leq C+\frac{q \gamma_{q}-p \gamma_{p}}{p} \int \mu_{1}\left|u_{n}\right|^{p} \leq C\left(1+\rho_{n}^{p \gamma_{p}}\right)
\end{aligned}
$$

which implies that $\left(u_{n}, v_{n}\right)$ is bounded.

Case-3: $\bar{p}<r \leq q$. From $Z_{n}\left(r \gamma_{r}\right) \leq C$, we get

$$
\frac{r \gamma_{r}-2}{2} \rho_{n}^{2} \leq C+\frac{r \gamma_{r}-p \gamma_{p}}{p} \int \mu_{1}\left|u_{n}\right|^{p} \leq C\left(1+\rho_{n}^{p \gamma_{p}}\right)
$$

which implies that $\left(u_{n}, v_{n}\right)$ is bounded.

Case-4: $\bar{p}<q<r$. From $Z_{n}\left(q \gamma_{q}\right) \leq C$, we get

$$
\frac{q \gamma_{q}-2}{2} \rho_{n}^{2} \leq C+\frac{q \gamma_{q}-p \gamma_{p}}{p} \int \mu_{1}\left|u_{n}\right|^{p} \leq C\left(1+\rho_{n}^{p \gamma_{p}}\right),
$$

which implies that $\left(u_{n}, v_{n}\right)$ is bounded.

Since the sequence $\left(u_{n}, v_{n}\right)$ is a bounded sequence of radial functions, by the compactness of the embed$\operatorname{ding} H_{r}^{1} \hookrightarrow L^{p}\left(\mathbb{R}^{N}\right)$ for $2<p<2^{*}$, there exists a $(u, v) \in H$ such that up to a subsequence $\left(u_{n}, v_{n}\right) \rightarrow(u, v)$ in $H$ and $L^{2^{*}}\left(\mathbb{R}^{N}\right) \times L^{2^{*}}\left(\mathbb{R}^{N}\right)$ and $\left(u_{n}, v_{n}\right) \rightarrow(u, v)$ in $L^{p}\left(\mathbb{R}^{N}\right) \times L^{p}\left(\mathbb{R}^{N}\right), L^{r}\left(\mathbb{R}^{N}\right) \times L^{r}\left(\mathbb{R}^{N}\right), L^{q}\left(\mathbb{R}^{N}\right) \times$ $L^{q}\left(\mathbb{R}^{N}\right)$ when $q<2^{*}$, and $\left(u_{n}, v_{n}\right) \rightarrow(u, v)$ a.e. in $\mathbb{R}^{N}$. Hence $u, v \geq 0$ are radial funtions. Since $\left.I\right|_{S_{a_{1}} \times S_{a_{2}}} ^{\prime}\left(u_{n}, v_{n}\right) \rightarrow 0$, by the Lagrange multiplier's rule, we have that there exists a sequence $\left(\lambda_{1, n}, \lambda_{2, n}\right) \subset$ $\mathbb{R}^{2}$ such that

$$
\begin{gathered}
\int_{\mathbb{R}^{N}} \nabla u_{n} \cdot \nabla \varphi+\lambda_{1, n} u_{n} \varphi-\mu_{1}\left|u_{n}\right|^{p-2} u_{n} \varphi-\beta r_{1}\left|u_{n}\right|^{r_{1}-2}\left|v_{n}\right|^{r_{2}} u_{n} \varphi=o(1)\|\varphi\|_{H^{1}}, \\
\int_{\mathbb{R}^{N}} \nabla v_{n} \cdot \nabla \psi+\lambda_{2, n} v_{n} \psi-\mu_{2}\left|v_{n}\right|^{q-2} v_{n} \psi-\beta r_{2}\left|u_{n}\right|^{r_{1}}\left|v_{n}\right|^{r_{2}-2} v_{n} \psi=o(1)\|\psi\|_{H^{1}},
\end{gathered}
$$

as $n \rightarrow \infty$, for every $(\varphi, \psi) \in H$. By choosing $(\varphi, \psi)=\left(u_{n}, v_{n}\right)$, we decude that $\left(\lambda_{1, n}, \lambda_{2, n}\right)$ is bounded as well, and hence up to a subsequence $\left(\lambda_{1, n}, \lambda_{2, n}\right) \rightarrow\left(\lambda_{1}, \lambda_{2}\right) \in \mathbb{R}^{2}$. Then, passing to the limits in (3.2)-3.3), we deduce that $(u, v)$ is a nonnegative solution of (1.2). Thus from the Pohozaev identity we obtain

$$
\lambda_{1}|u|_{2}^{2}+\lambda_{2}|v|_{2}^{2}=\left(1-\gamma_{p}\right) \int_{\mathbb{R}^{N}} \mu_{1} u^{p}+\left(1-\gamma_{q}\right) \int_{\mathbb{R}^{N}} \mu_{2} v^{q}+\left(1-\gamma_{r}\right) r \int_{\mathbb{R}^{N}} \beta u^{r_{1}} v^{r_{2}} .
$$

Moreover, combining $P\left(u_{n}, v_{n}\right) \rightarrow 0$ with 3.2) (3.3), we have

$$
\begin{aligned}
& \lambda_{1} a_{1}^{2}+\lambda_{2} a_{2}^{2}=\lim _{n \rightarrow \infty} \lambda_{1, n}\left|u_{n}\right|_{2}^{2}+\lambda_{2, n}\left|v_{n}\right|_{2}^{2} \\
= & \lim _{n \rightarrow \infty} \int_{\mathbb{R}^{N}}-\left(\left|\nabla u_{n}\right|^{2}+\left|\nabla v_{n}\right|^{2}\right)+\mu_{1}\left|u_{n}\right|^{p}+\mu_{2}\left|v_{n}\right|^{q}+r \beta\left|u_{n}\right|^{r_{1}}\left|v_{n}\right|^{r_{2}} \\
= & \lim _{n \rightarrow \infty}\left(1-\gamma_{p}\right) \int_{\mathbb{R}^{N}} \mu_{1}\left|u_{n}\right|^{p}+\left(1-\gamma_{q}\right) \int_{\mathbb{R}^{N}} \mu_{2}\left|v_{n}\right|^{q}+\left(1-\gamma_{r}\right) r \int_{\mathbb{R}^{N}} \beta\left|u_{n}\right|^{r_{1}}\left|v_{n}\right|^{r_{2}} \\
= & \left(1-\gamma_{p}\right) \int_{\mathbb{R}^{N}} \mu_{1} u^{p}+\left(1-\gamma_{q}\right) \int_{\mathbb{R}^{N}} \mu_{2} v^{q}+\left(1-\gamma_{r}\right) r \int_{\mathbb{R}^{N}} \beta u^{r_{1}} v^{r_{2}} .
\end{aligned}
$$

Now since there may be $u=0$ or $v=0$, we will proceed in four cases. 
Case 1: $u=0, v=0$. Since $\left(u_{n}, v_{n}\right) \rightarrow(u, v)$ in $L^{p}\left(\mathbb{R}^{N}\right) \times L^{p}\left(\mathbb{R}^{N}\right), L^{r}\left(\mathbb{R}^{N}\right) \times L^{r}\left(\mathbb{R}^{N}\right)$, we have

$$
0=P\left(u_{n}, v_{n}\right)+o(1)=\int_{\mathbb{R}^{N}}\left|\nabla u_{n}\right|^{2}+\left|\nabla v_{n}\right|^{2}-\gamma_{q} \int_{\mathbb{R}^{N}} \mu_{2}\left|v_{n}\right|^{q}+o(1) .
$$

Then it follows that

$$
\begin{aligned}
m\left(a_{1}, a_{2}\right) & =\lim _{n \rightarrow \infty} I\left(u_{n}, v_{n}\right)=\lim _{n \rightarrow \infty} \frac{1}{2} \int_{\mathbb{R}^{N}}\left|\nabla u_{n}\right|^{2}+\left|\nabla v_{n}\right|^{2}-\frac{1}{q} \int_{\mathbb{R}^{N}} \mu_{2}\left|v_{n}\right|^{q} \\
& =\lim _{n \rightarrow \infty}\left(\frac{1}{2}-\frac{1}{q \gamma_{q}}\right) \int_{\mathbb{R}^{N}}\left|\nabla u_{n}\right|^{2}+\left|\nabla v_{n}\right|^{2} \geq 0 .
\end{aligned}
$$

However, $m\left(a_{1}, a_{2}\right)<m\left(a_{1}, 0\right)<0$, we get a contradiction.

Case 2: $u \neq 0, v=0$. By the maximum principle, $u$ is a positive solution of 2.7 with parameters $p, \mu_{1}$ and $a=|u|_{2} \leq a_{1}$, then $m\left(a_{1}, 0\right) \leq m\left(|u|_{2}, 0\right)=I(u, 0)$. Let $\bar{u}_{n}=u_{n}-u$, then by using the Brezis-Lieb Lemma and Lemma 2.4, we have

$$
\begin{aligned}
0 & =P\left(u_{n}, v_{n}\right)+o(1)=P\left(\bar{u}_{n}, v_{n}\right)+P(u, 0)+o(1) \\
& =\int_{\mathbb{R}^{N}}\left|\nabla \bar{u}_{n}\right|^{2}+\left|\nabla v_{n}\right|^{2}-\gamma_{q} \int_{\mathbb{R}^{N}} \mu_{2}\left|v_{n}\right|^{q}+o(1),
\end{aligned}
$$

and hence

$$
\begin{aligned}
m\left(a_{1}, a_{2}\right) & =\lim _{n \rightarrow \infty} I\left(u_{n}, v_{n}\right)=\lim _{n \rightarrow \infty} I\left(\bar{u}_{n}, v_{n}\right)+I(u, 0) \\
& \geq \lim _{n \rightarrow \infty} \frac{1}{2} \int_{\mathbb{R}^{N}}\left|\nabla \bar{u}_{n}\right|^{2}+\left|\nabla v_{n}\right|^{2}-\frac{1}{q} \int_{\mathbb{R}^{N}} \mu_{2}\left|v_{n}\right|^{q}+m\left(a_{1}, 0\right) \\
& =\lim _{n \rightarrow \infty}\left(\frac{1}{2}-\frac{1}{q \gamma_{q}}\right) \int_{\mathbb{R}^{N}}\left|\nabla \bar{u}_{n}\right|^{2}+\left|\nabla v_{n}\right|^{2}+m\left(a_{1}, 0\right) \geq m\left(a_{1}, 0\right),
\end{aligned}
$$

which is a contradiction.

Case 3: $u=0, v \neq 0$. If $q=2^{*}$, then $v$ is a positive solution of 2.7 with parameters $p=2^{*}, \mu=\mu_{2}$ and $a=$ $|v|_{2}>0$, which contradicts Lemma2.2 If $q<2^{*}$, then proceeding as Case 2, we get $m\left(a_{1}, a_{2}\right) \geq m\left(0, a_{2}\right)$, a contradiction too.

Case 4: $u \neq 0, v \neq 0$. In this case, we prove $\left(u_{n}, v_{n}\right) \rightarrow(u, v)$ in $H$. Again by the maximum principle, $u, v>0$, then Lemma2.3 implies $\lambda_{1}, \lambda_{2}>0$. Moreover, from 3.4 (3.5), we obtain

$$
\lambda_{1}\left(a_{1}^{2}-|u|_{2}^{2}\right)+\lambda_{2}\left(a_{2}^{2}-|v|_{2}^{2}\right)=0
$$

and since $0<|u|_{2} \leq a_{1}, 0<|v|_{2} \leq a_{2}$ there must be $|u|_{2}=a_{1},|v|_{2}=a_{2}$. So $(u, v) \in \mathcal{P}_{a_{1}, a_{2}}$. Let $\left(\bar{u}_{n}, \bar{v}_{n}\right)=\left(u_{n}-u, v_{n}-v\right)$, then we have

$$
\begin{aligned}
0 & =P\left(u_{n}, v_{n}\right)+o(1)=P\left(\bar{u}_{n}, \bar{v}_{n}\right)+P(u, v)+o(1) \\
& =\int_{\mathbb{R}^{N}}\left|\nabla \bar{u}_{n}\right|^{2}+\left|\nabla \bar{v}_{n}\right|^{2}-\gamma_{q} \int_{\mathbb{R}^{N}} \mu_{2}\left|\bar{v}_{n}\right|^{q}+o(1),
\end{aligned}
$$


and hence

$$
\begin{aligned}
m\left(a_{1}, a_{2}\right) & =\lim _{n \rightarrow \infty} I\left(u_{n}, v_{n}\right)=\lim _{n \rightarrow \infty} I\left(\bar{u}_{n}, \bar{v}_{n}\right)+I(u, v) \\
& \geq \lim _{n \rightarrow \infty} \frac{1}{2} \int_{\mathbb{R}^{N}}\left|\nabla \bar{u}_{n}\right|^{2}+\left|\nabla \bar{v}_{n}\right|^{2}-\frac{1}{q} \int_{\mathbb{R}^{N}} \mu_{2}\left|\bar{v}_{n}\right|^{q}+m\left(a_{1}, a_{2}\right) \\
& =\lim _{n \rightarrow \infty}\left(\frac{1}{2}-\frac{1}{q \gamma_{q}}\right) \int_{\mathbb{R}^{N}}\left|\nabla \bar{u}_{n}\right|^{2}+\left|\nabla \bar{v}_{n}\right|^{2}+m\left(a_{1}, a_{2}\right) \geq m\left(a_{1}, a_{2}\right) .
\end{aligned}
$$

So $I(u, v)=m\left(a_{1}, a_{2}\right)$ and $\left(u_{n}, v_{n}\right) \rightarrow(u, v)$ in $H$.

Proof of the Theorem 1.1 Take $\alpha_{0}=\min \left\{\alpha_{1}, \alpha_{2}\right\}$, then by Lemma 3.4 and Lemma 3.5, it is sufficient to prove the existence of a radial Palais-Smale sequence for $\left.I\right|_{S_{a_{1}} \times S_{a_{2}}}$ at level $m\left(a_{1}, a_{2}\right)$ with additional properties $P\left(u_{n}, v_{n}\right) \rightarrow 0$ and $u_{n}^{-}, v_{n}^{-} \rightarrow 0$ a.e. in $\mathbb{R}^{N}$.

Let $m_{r}\left(a_{1}, a_{2}\right)=\inf _{A_{R_{0}} \cap H_{r}} I(u, v)$, and using the symmetric decreasing rearrangement it is easy to check $m\left(a_{1}, a_{2}\right)=m_{r}\left(a_{1}, a_{2}\right)$. Choosing a minimizing sequence $\left(\tilde{u}_{n}, \tilde{v}_{n}\right)$ for $m\left(a_{1}, a_{2}\right)=\inf _{A_{R_{0}} \cap H_{r}} I(u, v)$, we assume $\left(\tilde{u}_{n}, \tilde{v}_{n}\right)$ are nonnegative by replacing $\left(\tilde{u}_{n}, \tilde{v}_{n}\right)$ with $\left(\left|\tilde{u}_{n}\right|,\left|\tilde{v}_{n}\right|\right)$. Futhermore, using the fact that $I\left(s_{\left(\tilde{u}_{n}, \tilde{v}_{n}\right)} \star\left(\tilde{u}_{n}, \tilde{v}_{n}\right)\right) \leq I\left(\tilde{u}_{n}, \tilde{v}_{n}\right)$, and replacing $\left(\tilde{u}_{n}, \tilde{v}_{n}\right)$ by $s_{\left(\tilde{u}_{n}, \tilde{v}_{n}\right)} \star\left(\tilde{u}_{n}, \tilde{v}_{n}\right)$, we obtain a minimizing sequence $\left(\tilde{u}_{n}, \tilde{v}_{n}\right) \in \mathcal{P}_{a_{1}, a_{2}, r}^{+}$for $n \geq 1$. Therefore, by Ekeland's varational principle, there is a radial PalaisSmale sequence $\left(u_{n}, v_{n}\right)$ for $\left.I\right|_{S_{a_{1}, r} \times S_{a_{2}, r}}$ (hence a Palais-Smale sequence for $\left.I\right|_{S_{a_{1}} \times S_{a_{2}}}$ ) with the property $\left\|\left(u_{n}, v_{n}\right)-\left(\tilde{u}_{n}, \tilde{v}_{n}\right)\right\| \rightarrow 0$ as $n \rightarrow \infty$, which implies that

$$
P\left(u_{n}, v_{n}\right)=P\left(\tilde{u}_{n}, \tilde{v}_{n}\right)+o(1) \rightarrow 0 \text { and } u_{n}^{-}, v_{n}^{-} \rightarrow 0 \text { a.e. in } \mathbb{R}^{N},
$$

then we finish the proof.

\section{The purely $L_{2}$-supercritical case}

In this section, we suppose $\bar{p}<p, q, r<2^{*}$. To start our discussion, we consider once again the Pohozaev manifold $\mathcal{P}_{a_{1}, a_{2}}$ and the decomposition $\mathcal{P}_{a_{1}, a_{2}}=\mathcal{P}_{a_{1}, a_{2}}^{+} \cup \mathcal{P}_{a_{1}, a_{2}}^{0} \cup \mathcal{P}_{a_{1}, a_{2}}^{-}$. If there is a $(u, v) \in \mathcal{P}_{a_{1}, a_{2}}^{0}$, then combining $\Phi_{(u, v)}^{\prime}(0)=0$ and $\Phi_{(u, v)}^{\prime \prime}(0)=0$, we deduce that

$$
\left(p \gamma_{p}-2\right) \gamma_{p} \int_{\mathbb{R}^{N}} \mu_{1}|u|^{p}+\left(q \gamma_{q}-2\right) \int_{\mathbb{R}^{N}} \mu_{2}|v|^{q}+\left(r \gamma_{r}-2\right) r \gamma_{r} \int_{\mathbb{R}^{N}} \beta|u|^{r_{1}}|v|^{r_{2}}=0 .
$$

Since $p \gamma_{p}, r \gamma_{r}, q \gamma_{q}>2$, there must be $(u, v)=(0,0)$, in contradiction with $(u, v) \in S_{a_{1}} \times S_{a_{2}}$. This shows that $\mathcal{P}_{a_{1}, a_{2}}^{0}=\emptyset$, and then as Lemma 3.2 we can prove that $\mathcal{P}_{a_{1}, a_{2}}$ is a $C^{1}$ submanifold in $H$ with codimension 3. However, in this section, the geometry of $\mathcal{P}_{a_{1}, a_{2}}$ will be different from the one in Lemma3.3

Lemma 4.1. For any $(u, v) \in S_{a_{1}} \times S_{a_{2}}$, the function $\Phi_{(u, v)}$ has a unique critical point $t_{(u, v)} \in \mathbb{R}$, which is a strict maximum point at positive level. Moreover,

(1) $\mathcal{P}_{a_{1}, a_{2}}=\mathcal{P}_{a_{1}, a_{2}}^{-}$and $P(u, v)<0$ iff $t_{(u, v)}<0$;

(2) $\Phi_{(u, v)}$ is strict increasing in $\left(-\infty, t_{(u, v)}\right)$;

(3) the map $(u, v) \mapsto t_{(u, v)}$ is of class $C^{1}$.

Proof. The proof is completely the sames as [22, Lemma 6.1], and we omit the details here. 
Using the above lemma, it is easy to see that

$$
m\left(a_{1}, a_{2}\right)=\inf _{S_{a_{1}} \times S_{a_{2}}} \max _{t \in \mathbb{R}} I(t \star(u, v)) .
$$

By using the same techniques as that in Lemma3.5, we can prove the following lemma.

Lemma 4.2. Suppose $N=3,4$. Let $\left(u_{n}, v_{n}\right) \subset S_{a_{1}} \times S_{a_{2}}$ be a radial Palais-Smale sequence for $\left.I\right|_{S_{a_{1}} \times S_{a_{2}}}$ at level $m\left(a_{1}, a_{2}\right)$ with the additional properties $P\left(u_{n}, v_{n}\right) \rightarrow 0$ and $u_{n}^{-}, v_{n}^{-} \rightarrow 0$ a.e. in $\mathbb{R}^{N}$. If

$$
0<m\left(a_{1}, a_{2}\right)<\min \left\{m\left(a_{1}, 0\right), m\left(0, a_{2}\right)\right\},
$$

then up to a subsequence $\left(u_{n}, v_{n}\right) \rightarrow(u, v)$ in $H$, where $(u, v)$ is a positive solution of (1.2) for some $\lambda_{1}, \lambda_{2}>$ 0 .

Remark 4.1. It is natural that $m\left(a_{1}, a_{2}\right)>0$. Indeed, for any $(u, v) \in \mathcal{P}_{a_{1}, a_{2}}$, there is

$$
\begin{aligned}
\int_{\mathbb{R}^{N}}|\nabla u|^{2}+|\nabla v|^{2}= & \gamma_{p} \int_{\mathbb{R}^{N}} \mu_{1}|u|^{p}+\gamma_{q} \int_{\mathbb{R}^{N}} \mu_{2}|v|^{q}+r \gamma_{r} \int_{\mathbb{R}^{N}} \beta|u|^{r_{1}}|v|^{r_{2}} \\
\leq & \mathcal{D}_{2} p \gamma_{p}\left(\int_{\mathbb{R}^{N}}|\nabla u|^{2}\right)^{\frac{p \gamma_{p}}{2}}+\mathcal{D}_{3} q \gamma_{q}\left(\int_{\mathbb{R}^{N}}|\nabla v|^{2}\right)^{\frac{q \gamma_{q}}{2}} \\
& +\mathcal{D}_{1} r \gamma_{r} \beta\left(\int_{\mathbb{R}^{N}}|\nabla u|^{2}+|\nabla v|^{2}\right)^{\frac{r \gamma_{r}}{2}}
\end{aligned}
$$

which implies $\inf _{\mathcal{P}_{a_{1}, a_{2}}} \int|\nabla u|^{2}+|\nabla v|^{2} \geq C>0$. So we have

$$
\begin{aligned}
& m\left(a_{1}, a_{2}\right)=\inf _{\mathcal{P}_{a_{1}, a_{2}}} I(u, v) \\
= & \inf _{\mathcal{P}_{a_{1}, a_{2}}} \frac{p \gamma_{p}-2}{2 p} \int_{\mathbb{R}^{N}} \mu_{1}|u|^{p}+\frac{q \gamma_{q}-2}{2 q} \int_{\mathbb{R}^{N}} \mu_{2}|u|^{q}+\frac{r \gamma_{r}-2}{2} \int_{\mathbb{R}^{N}} \beta|u|^{r_{1}}|v|^{r_{2}} \\
\geq & C \inf _{\mathcal{P}_{a_{1}, a_{2}}} \int_{\mathbb{R}^{N}}|\nabla u|^{2}+|\nabla v|^{2}>0 .
\end{aligned}
$$

We recall the following lemma in [3].

Lemma 4.3. The map $(s, u) \in \mathbb{R} \times H^{1} \rightarrow s \star u \in H^{1}$ is continuous.

Now we give a way to find such a Palais-Smale sequence as the required one in Lemma 4.2

Lemma 4.4. There is a radial Palais-Smale sequence for $\left.I\right|_{S_{a_{1}} \times S_{a_{2}}}$ at level $m\left(a_{1}, a_{2}\right)$ with the additional properties $P\left(u_{n}, v_{n}\right) \rightarrow 0$ and $u_{n}^{-}, v_{n}^{-} \rightarrow 0$ a.e. in $\mathbb{R}^{N}$.

Proof. We consider the functional $\tilde{I}: \mathbb{R} \times H^{1}\left(\mathbb{R}^{N}\right) \times H^{1}\left(\mathbb{R}^{N}\right) \rightarrow \mathbb{R}$ defined by

$$
\tilde{I}(s, u, v):=I(s \star(u, v))
$$

on the constraint $\mathbb{R} \times S_{a_{1}, r} \times S_{a_{2}, r}$. Denote the closed sublevel set by $I^{c}=\left\{(u, v) \in S_{a_{1}} \times S_{a_{2}}: I(u, v) \leq c\right\}$. We note that for any $(u, v) \in S_{a_{1}} \times S_{a_{2}}$,

$$
I(u, v) \geq \frac{1}{2}\left(|\nabla u|_{2}^{2}+|\nabla v|_{2}^{2}\right)-\mathcal{D}_{2}|\nabla u|_{2}^{p \gamma_{p}}-\mathcal{D}_{3}|\nabla v|_{2}^{q \gamma_{q}}-\mathcal{D}_{1} \beta\left(|\nabla u|_{2}^{2}+|\nabla v|_{2}^{2}\right)^{\frac{r \gamma_{r}}{2}},
$$




$$
\begin{gathered}
I(u, v) \leq \frac{1}{2}\left(|\nabla u|_{2}^{2}+|\nabla v|_{2}^{2}\right), \\
P(u, v) \geq|\nabla u|_{2}^{2}+|\nabla v|_{2}^{2}-\mathcal{D}_{1} p \gamma_{p}|\nabla u|_{2}^{p \gamma_{p}}-\mathcal{D}_{3} q \gamma_{q}|\nabla v|_{2}^{q \gamma_{q}}-\mathcal{D}_{1} r \gamma_{r} \beta\left(|\nabla u|_{2}^{2}+|\nabla v|_{2}^{2}\right)^{\frac{r \gamma_{r}}{2}},
\end{gathered}
$$

then there exists a small $k>0$ such that

$$
0<I(u, v)<m\left(a_{1}, a_{2}\right), \quad P(u, v)>0, \quad \forall(u, v) \in \bar{A}_{k} .
$$

We introduce the minimax class

$$
\Gamma:=\left\{\gamma=\left(\alpha, \varphi_{1}, \varphi_{2}\right) \in C\left([0,1], \mathbb{R} \times S_{a_{1}, r} \times S_{a_{2}, r}\right): \gamma(0) \in\{0\} \times \bar{A}_{k}, \gamma(1) \in\{0\} \times I^{0}\right\}
$$

with the associated minimax level

$$
\sigma:=\inf _{\gamma \in \Gamma} \max _{t \in[0,1]} \tilde{I}(\gamma(t)) .
$$

Next we check that $\sigma=m\left(a_{1}, a_{2}\right)$. On the one hand, for any $(u, v) \in \mathcal{P}_{a_{1}, a_{2}}$, there are $\left(u^{*}, v^{*}\right) \in S_{a_{1}, r} \times S_{a_{2}, r}$ and $P\left(u^{*}, v^{*}\right) \leq P(u, v)=0$, which implies $t_{*}=t_{\left(u^{*}, v^{*}\right)} \leq 0$. It follows that

$$
I(u, v) \geq I\left(t_{*} \star(u, v)\right) \geq I\left(t_{*} \star\left(u^{*}, v^{*}\right)\right)=\max _{t \in \mathbb{R}} I\left(t \star\left(u^{*}, v^{*}\right)\right) .
$$

Observing that

$$
\begin{gathered}
\left|\nabla s \star u^{*}\right|_{2}^{2}+\left|\nabla s \star v^{*}\right|_{2}^{2} \rightarrow 0, \quad \text { as } s \rightarrow-\infty, \\
I\left(t \star\left(u^{*}, v^{*}\right)\right) \rightarrow-\infty, \quad \text { as } s \rightarrow \infty,
\end{gathered}
$$

we choose $s_{0} \ll-1, s_{1} \gg 1$ such that $s_{0} \star\left(u^{*}, v^{*}\right) \in A_{k}$ and $s_{1} \star\left(u^{*}, v^{*}\right) \in I^{0}$. Then we define $\gamma_{*}:[0,1] \rightarrow$ $\mathbb{R} \times S_{a_{1}, r} \times S_{a_{2}, r}$

$$
\gamma_{*}(t)=\left(0,\left[(1-t) s_{0}+t s_{1}\right] \star\left(u^{*}, v^{*}\right)\right),
$$

and by Lemma4.3, $\gamma_{*} \in \Gamma$. Hence

$$
\sigma \leq \max _{t \in[0,1]} \tilde{I}\left(\gamma_{*}(t)\right) \leq \max _{t \in \mathbb{R}} I\left(t \star\left(u^{*}, v^{*}\right)\right) \leq I(u, v),
$$

which implies $\sigma \leq m\left(a_{1}, a_{2}\right)$. On the other hand, for any $\gamma=\left(\alpha, \varphi_{1}, \varphi_{2}\right) \in \Gamma$, we consider the function

$$
P_{\gamma}: t \in[0,1] \rightarrow P\left(\alpha(t) \star\left(\varphi_{1}(t), \varphi_{2}(t)\right)\right) \in \mathbb{R} .
$$

It is easy to see that $P_{\gamma}$ is continuous and $P_{\gamma}(0)>0$. We claim that $P_{\gamma}(1)<0$. Indeed, if $P_{\gamma}(1) \geq 0$, we have $t_{\left(\varphi_{1}(1), \varphi_{2}(1)\right)} \geq 0$, and then from Lemma4.1.

$$
I\left(\varphi_{1}(1), \varphi_{2}(1)\right)=\Phi_{\left(\varphi_{1}(1), \varphi_{2}(1)\right)}(0)>\Phi_{\left(\varphi_{1}(1), \varphi_{2}(1)\right)}(-\infty)=0^{+},
$$

which is a contradiction. Thus we obtain a $t_{\gamma} \in(0,1)$ such that $P_{\gamma}\left(t_{\gamma}\right)=0$. It follows that

$$
\max _{t \in[0,1]} \tilde{I}(\gamma(t)) \geq \tilde{I}\left(\gamma\left(t_{\gamma}\right)\right)=I\left(\alpha\left(t_{\gamma}\right) \star\left(\varphi_{1}\left(t_{\gamma}\right), \varphi_{2}\left(t_{\gamma}\right)\right)\right) \geq m\left(a_{1}, a_{2}\right),
$$

which implies $\sigma \geq m\left(a_{1}, a_{2}\right)$. Hence $\sigma=m\left(a_{1}, a_{2}\right)$.

Let $\mathcal{F}=\{\gamma([0,1]): \gamma \in \Gamma\}$. Using the terminology in [9, Section 5], $\mathcal{F}$ is a homotopy stable family of compact subset of $\mathbb{R} \times S_{a_{1}, r} \times S_{a_{2}, r}$ with extended closed boundary $\{0\} \times \bar{A}_{k} \cup\{0\} \times I^{0}$, and the superlevel set $\{\tilde{I} \geq \sigma\}$ is a dual set for $\mathcal{F}$, which means that the assumptions in [9, Theorem 5.2] are satisfied. Therefore, taking a minimizing sequence $\left\{\gamma_{n}([0,1]), \gamma_{n}=\left(\alpha_{n}, \varphi_{1, n}, \varphi_{2, n}\right)\right\}$ for $\sigma$ with the property that $\alpha(t)=0$, 
$\varphi_{1, n}(t) \geq 0, \varphi_{2, n}(t) \geq 0$ for every $t \in[0,1]$ ( Indeed, we can replace $\gamma_{n}$ by $\left.\tilde{\gamma}_{n}=\left(0, \alpha_{n} \star\left(\left|\varphi_{1, n}\right|,\left|\varphi_{2, n}\right|\right)\right)\right)$, there exists a sequence $\left(s_{n}, u_{n}, v_{n}\right) \subset \mathbb{R} \times S_{a_{1}, r} \times S_{a_{2}, r}$ such that as $n \rightarrow \infty, \tilde{I}\left(s_{n}, u_{n}, v_{n}\right) \rightarrow \sigma$ and

$$
\begin{gathered}
\partial_{s} \tilde{I}\left(s_{n}, u_{n}, v_{n}\right) \rightarrow 0, \quad\left\|\partial_{(u, v)} \tilde{I}\left(s_{n}, u_{n}, v_{n}\right)\right\|_{T_{u_{n}} S_{a_{1}, r} \times T_{v_{n}} S_{a_{2}, r}} \rightarrow 0, \\
\left|s_{n}\right|+\operatorname{dist}\left(\left(u_{n}, v_{n}\right),\left(\varphi_{1, n}([0,1]), \varphi_{2, n}([0,1])\right)\right) \rightarrow 0 .
\end{gathered}
$$

Let $\left(\bar{u}_{n}, \bar{v}_{n}\right)=s_{n} \star\left(u_{n}, v_{n}\right) \in S_{a_{1}, r} \times S_{a_{2}, r}$. From 4.2 , we know that $\left\{s_{n}\right\}$ is bounded and $\bar{u}_{n}^{-}, \bar{v}_{n}^{-} \rightarrow 0$ a.e. in $\mathbb{R}^{N}$. Moreover, 4.1] implies that

$$
P\left(\bar{u}_{n}, \bar{v}_{n}\right)=\partial_{s} \tilde{I}\left(s_{n}, u_{n}, v_{n}\right) \rightarrow 0,
$$

and that

$$
\begin{aligned}
I^{\prime}\left(\bar{u}_{n}, \bar{v}_{n}\right)[\phi, \psi] & =\partial_{(u, v)} \tilde{I}\left(s_{n}, u_{n}, v_{n}\right)\left[\left(-s_{n}\right) \star(\phi, \psi)\right] \\
& =o(1)\left\|\left(-s_{n}\right) \star(\phi, \psi)\right\|_{H} \\
& =o(1)\|(\phi, \psi)\|_{H},
\end{aligned}
$$

for any $(\phi, \psi) \in T_{\bar{u}_{n}} S_{a_{1}, r} \times T_{\bar{v}_{n}} S_{a_{2}, r}$. Summing up, $\left(\bar{u}_{n}, \bar{v}_{n}\right)$ is a radial Palais-Smale sequence of $\left.I\right|_{S_{a_{1}}^{r} \times S_{a_{2}}^{r}}$ and hence a radial symmetric Palais-Smale sequence of $\left.I\right|_{S_{a_{1}} \times S_{a_{2}}}$ at level $\sigma$.

Before giving an estimate of $m\left(a_{1}, a_{2}\right)$ from above, we would like to study the dependence of $m\left(a_{1}, a_{2}\right)$ on $\beta$. In the following lemma, we denote $m\left(a_{1}, a_{2}\right), I(u, v)$ by $m_{\beta}\left(a_{1}, a_{2}\right), I_{\beta}(u, v)$ respectively.

Lemma 4.5. For any $a_{1}, a_{2}>0$, we have that

(1) $m_{\beta}\left(a_{1}, a_{2}\right)$ is decreasing with respect to $\beta \geq 0$;

(2) $m_{0}\left(a_{1}, a_{2}\right)=\min \left\{m\left(a_{1}, 0\right), m\left(0, a_{2}\right)\right\}$.

Proof. (1) For any $\beta_{1} \geq \beta_{2} \geq 0$,

$$
m_{\beta_{1}}\left(a_{1}, a_{2}\right)=\inf _{S_{a_{1}} \times S_{a_{2}}} \max _{t \in \mathbb{R}} I_{\beta_{1}}(t \star(u, v)) \leq \inf _{S_{a_{1}} \times S_{a_{2}}} \max _{t \in \mathbb{R}} I_{\beta_{2}}(t \star(u, v))=m_{\beta_{2}}\left(a_{1}, a_{2}\right),
$$

so $m_{\beta}\left(a_{1}, a_{2}\right)$ is decreasing with respect to $\beta \geq 0$.

(2) Let $l=\min \left\{m\left(a_{1}, 0\right), m\left(0, a_{2}\right)\right\}$. We first prove $m_{0}\left(a_{1}, a_{2}\right) \geq l$. Suppose $0<m_{0}\left(a_{1}, a_{2}\right)<l$. Then by Lemma 4.2 and Lemma 4.4 we can find a sequence $\left(u_{n}, v_{n}\right) \rightarrow\left(u_{0}, v_{0}\right)$ in $H$, where $\left(u_{0}, v_{0}\right)$ attains the minimum problem $m_{0}\left(a_{1}, a_{2}\right)$. Since $\beta=0$, the system (1.3) is given by two uncoupled equations and both $u_{0}$ and $v_{0}$ are positive radial solutions. By Lemma2.1, we have

$$
l>m_{0}\left(a_{1}, a_{2}\right)=I_{0}\left(u_{0}, v_{0}\right)=m\left(a_{1}, 0\right)+m\left(0, a_{2}\right)>l,
$$

a contradiction.

Now we prove $m_{0}\left(a_{1}, a_{2}\right) \leq l$. Let $u$ be the unique positive solution of (2.7) with parameters $p, \mu_{1}, a_{1}$ and $v$ be the unique positive solution of (2.7) with parameters $q, \mu_{2}, a_{2}$. So $(u, v) \in S_{a_{1}} \times S_{a_{2}}$ and $(u, s \star v) \in$ $S_{a_{1}} \times S_{a_{2}}$ for any $s \in \mathbb{R}$. Let $t_{s}=t_{(u, s \star v)}$, there is

$$
\begin{aligned}
0 & =P_{0}\left(t_{s} \star(u, s \star v)\right) \\
& =e^{2 t_{s}} \int_{\mathbb{R}^{N}}|\nabla u|^{2}+e^{2 t_{s}+2 s} \int_{\mathbb{R}^{N}}|\nabla v|^{2}-e^{p \gamma_{p} t_{s}} \int_{\mathbb{R}^{N}} \mu_{1}|u|^{p}-e^{q \gamma_{q}\left(t_{s}+s\right)} \int_{\mathbb{R}^{N}} \mu_{2}|v|^{q},
\end{aligned}
$$


which means that

$$
\int_{\mathbb{R}^{N}}|\nabla u|^{2}+e^{2 s} \int_{\mathbb{R}^{N}}|\nabla v|^{2} \geq e^{\left(p \gamma_{p}-2\right) t_{s}} \int_{\mathbb{R}^{N}} \mu_{1}|u|^{p} .
$$

Therefore, $e^{t_{s}}$ is bounded as $s \rightarrow-\infty$. Hence, for any $s \in \mathbb{R}$,

$$
\begin{aligned}
m_{0}\left(a_{1}, a_{2}\right) & \leq I_{0}\left(t_{s} \star(u, s \star v)\right)=E_{p, \mu_{1}}\left(t_{s} \star u\right)+E_{q, \mu_{2}}\left(\left(t_{s}+s\right) \star v\right) \\
& \leq m\left(a_{1}, 0\right)+\frac{e^{2\left(t_{s}+s\right)}}{2} \int_{\mathbb{R}^{N}}|\nabla u|^{2}-\frac{e^{q \gamma_{q}\left(t_{s}+s\right)}}{q} \int_{\mathbb{R}^{N}} \mu_{2}|v|^{q} .
\end{aligned}
$$

Let $s \rightarrow-\infty$, we obtain $m_{0}\left(a_{1}, a_{2}\right) \leq m\left(a_{1}, 0\right)$. Similarly we can prove that $m_{0}\left(a_{1}, a_{2}\right) \leq m\left(0, a_{2}\right)$.

Lemma 4.6. For any $a_{1}, a_{2}>0$, we have that

(1) there exists $a \beta_{0}>0$ such that $m\left(a_{1}, a_{2}\right)<\min \left\{m\left(a_{1}, 0\right), m\left(0, a_{2}\right)\right\}$ for any $\beta>\beta_{0}$;

(2) futher, if $r_{1}, r_{2}<2$, then $m\left(a_{1}, a_{2}\right)<\min \left\{m\left(a_{1}, 0\right), m\left(0, a_{2}\right)\right\}$ for any $\beta>0$.

Proof. (1) Let $u$ be the unique positive solution of (2.7) with parameters $p, \mu_{1}, a_{1}$ and $v$ be the unique positive solution of 2.77 with parameters $q, \mu_{2}, a_{2}$. It is easy to see that

$$
E_{p, \mu_{1}}(s \star u) \rightarrow 0 \text { and } \quad E_{q, \mu_{2}}(s \star v) \rightarrow 0 \quad \text { as } s \rightarrow-\infty .
$$

So there exists a $s_{0}<-1$ which is independent of $\beta$ such that

$$
\max _{s<s_{0}} I(s \star(u, v))<\max _{s<s_{0}} E_{p, \mu_{1}}(s \star u)+E_{q, \mu_{2}}(s \star v)<\min \left\{m\left(a_{1}, 0\right), m\left(0, a_{2}\right)\right\} .
$$

If $s \geq s_{0}$, then the intersection term can be bounded from below:

$$
\int_{\mathbb{R}^{N}}|s \star u|^{r_{1}}|s \star v|^{r_{2}}=e^{r \gamma_{r} s} \int_{\mathbb{R}^{N}}|u|^{r_{1}}|v|^{r_{2}} \geq C e^{r \gamma_{r} s_{0}} .
$$

As a consequence, we have

$$
\begin{aligned}
\max _{s \geq s_{0}} I(s \star(u, v)) & \leq \max _{s \geq s_{0}} E_{p, \mu_{1}}(s \star u)+E_{q, \mu_{2}}(s \star v)-C e^{r \gamma_{r} s_{0}} \beta \\
& \leq m\left(a_{1}, 0\right)+m\left(0, a_{2}\right)-C e^{r \gamma_{r} s_{0}} \beta,
\end{aligned}
$$

and the last term is strictly smaller than $\min \left\{m\left(a_{1}, 0\right), m\left(0, a_{2}\right)\right\}$ provided $\beta$ is sufficiently large.

(2) Let $\left(u, \lambda_{0}\right) \in S_{a_{1}} \times \mathbb{R}^{+}$be the unique positive solution in Lemma 2.1 with parameters $p, \mu_{1}, a_{1}$. Since $r_{2}<2$, we take a $m \in\left(\frac{N}{2}-\frac{2}{r_{2}}, \frac{N}{2}-1\right)$ and $v(x)=c \frac{\varphi(x)}{|x|^{m}}$ with

$$
\varphi(x) \in C_{0}^{\infty}\left(B_{2}(0)\right), \quad 0 \leq \varphi(x) \leq 1, \quad \varphi(x)=1 \text { in } B_{1}(0) .
$$

Then $v \in H$ and we choose a suitable $c$ such that $v \in S_{a_{2}}$. Therefore $(u, s \star v) \in S_{a_{1}} \times S_{a_{2}}$ for any $s \in \mathbb{R}$. Let

$$
\alpha(s)=\int_{\mathbb{R}^{N}}|u|^{r_{1}}|s \star v|^{r_{2}}=C_{0} e^{\left(\frac{N}{2}-m\right) r_{2} s} \int_{\mathbb{R}^{N}} u^{r_{1}}(x) \frac{\varphi^{r_{2}}\left(e^{s} x\right)}{|x|^{m r_{2}}} .
$$

As in Lemma 3.4 we can prove that

$$
\alpha(s)=C_{0} e^{\theta s}\left(C_{1}+o(1)\right)>\frac{C_{0} C_{1}}{2} e^{\theta s} \quad \text { as } s \rightarrow-\infty .
$$


where $C_{1}=\int_{\mathbb{R}^{N}} \frac{u^{r_{1}}(x)}{|x|^{m r_{2}}} \in(0, \infty)$ and $\theta=\left(\frac{N}{2}-m\right) r_{2} \in(1,2)$. Now let $t_{s}=t_{(u, s \star v)}$, then

$$
\begin{aligned}
0= & P_{0}\left(t_{s} \star(u, s \star v)\right) \\
= & e^{2 t_{s}} \int_{\mathbb{R}^{N}}|\nabla u|^{2}+e^{2 t_{s}+2 s} \int_{\mathbb{R}^{N}}|\nabla v|^{2}-e^{p \gamma_{p} t_{s}} \int_{\mathbb{R}^{N}} \mu_{1}|u|^{p} \\
& -e^{q \gamma_{q}\left(t_{s}+s\right)} \int_{\mathbb{R}^{N}} \mu_{2}|v|^{q}-\beta r \gamma_{r} e^{r \gamma_{r} t_{s}} \alpha(s),
\end{aligned}
$$

from which we obtain that there exists $C_{2}, C_{3}>0$ such that

$$
C_{2} \leq e^{t_{s}} \leq C_{3} \text { as } s \rightarrow-\infty .
$$

Without loss of generality, we assume $e^{t_{s}} \rightarrow l>0$ as $s \rightarrow-\infty$, then let $s \rightarrow-\infty$ in 4.4 , we conclude

$$
l^{2} \int_{\mathbb{R}^{N}}|\nabla u|^{2}-l^{p \gamma_{p}} \int_{\mathbb{R}^{N}} \mu_{1}|u|^{p}=0
$$

which menas $l=1$. Therefore

$$
\begin{aligned}
m\left(a_{1}, a_{2}\right) & \leq I\left(t_{s} \star(u, s \star v)\right) \\
& =E_{p, \mu_{1}}\left(t_{s} \star u\right)+\frac{e^{2\left(t_{s}+s\right)}}{2} \int_{\mathbb{R}^{N}}|\nabla v|^{2}-\frac{e^{q \gamma_{q}\left(t_{s}+s\right)}}{q} \int_{\mathbb{R}^{N}} \mu_{2}|v|^{q}-\beta e^{r \gamma_{r} t_{s}} \alpha(s) \\
& <m\left(a_{1}, 0\right)+\frac{e^{2\left(t_{s}+s\right)}}{2} \int_{\mathbb{R}^{N}}|\nabla v|^{2}-\frac{e^{q \gamma_{q}\left(t_{s}+s\right)}}{q} \int_{\mathbb{R}^{N}} \mu_{2}|v|^{q}-\beta e^{r \gamma_{r} t_{s}} \frac{C_{0} C_{1}}{2} e^{\theta s},
\end{aligned}
$$

from which, we see for sufficiently small $s \ll-1$, there holds $m\left(a_{1}, a_{2}\right)<m\left(a_{1}, 0\right)$. Similarly we can prove $m\left(a_{1}, a_{2}\right)<m\left(0, a_{2}\right)$.

Proof of the Theorem 1.2 The proof is finished when we combine Lemma 4.2. Lemma4.4 and Lemma 4.6

\section{A Proof of Lemma 3.1 and Remark 3.1}

Proof of Lemma 3.1 Since the monotonicity of $h(t)$ will be strongly affected by the comparision of $p, q$ and $r$, we need to divide the proof into four different situations.

Case 1: $p \leq r<\bar{p}$. We have $p \gamma_{p} \leq r \gamma_{r}<2<q \gamma_{q}$ and

$$
h^{\prime}(t)=t^{p \gamma_{p}-1}\left(t^{2-p \gamma_{p}}-\mathcal{D}_{1} \beta r \gamma_{r} t^{r \gamma_{r}-p \gamma_{p}}-\mathcal{D}_{2} p \gamma_{p}-\mathcal{D}_{3} q \gamma_{q} t^{t \gamma_{q}-p \gamma_{p}}\right) .
$$

Denote $g(t)=t^{2-p \gamma_{p}}-\mathcal{D}_{1} \beta r \gamma_{r} t^{r \gamma_{r}-p \gamma_{p}}-\mathcal{D}_{3} q \gamma_{q} t^{q \gamma_{q}-p \gamma_{p}}$, there are

$$
\begin{gathered}
h^{\prime}(t)=t^{p \gamma_{p}-1}\left(g(t)-\mathcal{D}_{2} p \gamma_{p}\right), \\
g^{\prime}(t)=t^{r \gamma_{r}-p \gamma_{p}-1}\left[\left(2-p \gamma_{p}\right) t^{2-r \gamma_{r}}-\mathcal{D}_{1} \beta r \gamma_{r}\left(r \gamma_{r}-p \gamma_{p}\right)-\mathcal{D}_{3} q \gamma_{q}\left(q \gamma_{q}-p \gamma_{p}\right) t^{q \gamma_{q}-r \gamma_{r}}\right] .
\end{gathered}
$$

Let $f(t)=\left(2-p \gamma_{p}\right) t^{2-r \gamma_{r}}-\mathcal{D}_{3} q \gamma_{q}\left(q \gamma_{q}-p \gamma_{p}\right) t^{q \gamma_{p}-r \gamma_{r}}$, then

$$
g^{\prime}(t)=t^{r \gamma_{r}-p \gamma_{p}-1}\left[f(t)-\mathcal{D}_{1} \beta r \gamma_{r}\left(r \gamma_{r}-p \gamma_{p}\right)\right],
$$




$$
f^{\prime}(t)=t^{1-r \gamma_{r}}\left[\left(2-p \gamma_{p}\right)\left(2-r \gamma_{r}\right)-\mathcal{D}_{3} q \gamma_{q}\left(q \gamma_{q}-p \gamma_{p}\right)\left(q \gamma_{q}-r \gamma_{r}\right) t^{q \gamma_{q}-2}\right] .
$$

Since $p \gamma_{p} \leq r \gamma_{r}<2<q \gamma_{q}$, we get $f(+\infty)=g(+\infty)=h(+\infty)=-\infty, f(0+)=0^{+}, h(0+)=0^{-}$and

$$
g(0+)=\left\{\begin{array}{l}
0^{-}, \quad p<r, \\
-\mathcal{D}_{1} \beta r \gamma_{r}<0, \quad p=r .
\end{array}\right.
$$

We see that $f(t)$ has a unique critical point $\bar{t}$ in $(0,+\infty)$ satisfying

$$
\bar{t}^{q \gamma_{q}-2}=\frac{2-p \gamma_{p}}{q \gamma_{q}-p \gamma_{p}} \frac{2-r \gamma_{r}}{q \gamma_{q}-r \gamma_{r}} \frac{1}{\mathcal{D}_{3} q \gamma_{q}} .
$$

Moreover, if

$$
\left.f(\bar{t})>\mathcal{D}_{1} \beta r \gamma_{r}\left(r \gamma_{r}-p \gamma_{p}\right)\right), \quad g(\bar{t})>\mathcal{D}_{2} p \gamma_{p}, \quad h(\bar{t})>0,
$$

then the function $h(t)$ has exactly two critical points, one is a local minimum at negitive level, the other one is a global maximum at positive level. Futher, there exists $0<R_{0}<R_{1}$ such that $h\left(R_{0}\right)=h\left(R_{1}\right)=0$, and $h(t)>0$ iff $t \in\left(R_{0}, R_{1}\right)$. On the other hand, from the definitions of $f(t), g(t)$ and $h(t)$, we can check that (A.2) is equivalent to

$$
\left\{\begin{array}{l}
\left.\left(2-p \gamma_{p}\right) \bar{t}^{2}>\mathcal{D}_{1} \beta r \gamma_{r}\left(r \gamma_{r}-p \gamma_{p}\right)\right) \bar{t}^{r \gamma_{r}}+\mathcal{D}_{3} q \gamma_{q}\left(q \gamma_{q}-p \gamma_{p}\right) \bar{t}^{q \gamma_{q}} \\
\bar{t}^{2}>\mathcal{D}_{1} \beta r \gamma_{r} \bar{t}^{r \gamma_{r}}+\mathcal{D}_{2} p \gamma_{p} \bar{t}^{p \gamma_{p}}+\mathcal{D}_{3} q \gamma_{q} \bar{t}^{q \gamma_{q}} \\
\frac{1}{2} \bar{t}^{2}>\mathcal{D}_{1} \beta \bar{t}^{r} \gamma_{r}+\mathcal{D}_{2} \bar{t}^{p \gamma_{p}}+\mathcal{D}_{3} \bar{t}^{q \gamma_{q}}
\end{array}\right.
$$

Substituting A.1 into A.3, we obtain a constant $C>0$ such that if

$$
\mathcal{D}_{1} \beta \mathcal{D}_{3}^{\frac{2-\gamma \gamma_{r}}{q \gamma_{q}-2}}+\mathcal{D}_{2} \mathcal{D}_{3}^{\frac{2-p \gamma_{p}}{q \gamma_{q}-2}}<C,
$$

then A.3 holds, which menas A.2 holds. It follows from the definitions of $\mathcal{D}_{1}, \mathcal{D}_{2}$ and $\mathcal{D}_{3}$ that we can immediately obtain a constant $\alpha_{1}$ with the required properties.

Case 2: $r<p<\bar{p}$. If we exchange the roles played by $\mathcal{D}_{2} t^{p \gamma_{p}}$ and $\mathcal{D}_{1} t^{r \gamma_{r}}$, then we can get the constant $\alpha_{1}$ as Case 1.

Case 3: $r=\bar{p}$. We first suppose $\alpha_{1}<\frac{1}{4}$, then $\delta:=\frac{1}{2}-\mathcal{D}_{1} \beta \in\left(\frac{1}{4}, \frac{1}{2}\right)$ when $\mathcal{D}_{1} \beta<\alpha_{1}$ and $h(t)$ turns to be

$$
h(t)=\delta t^{2}-\mathcal{D}_{2} t^{p \gamma_{p}}-\mathcal{D}_{3} t^{q \gamma_{q}} .
$$

Taking a similar argument as in Case 1, we can prove the existence of the constant $\alpha_{1}$.

Case 4: $r>\bar{p}$. Note that in this case $p \gamma_{p}<2<r \gamma_{r}, q \gamma_{q}$. Similarly we have

$$
h^{\prime}(t)=t^{p \gamma_{p}-1}\left(t^{2-p \gamma_{p}}-\mathcal{D}_{1} \beta r \gamma_{r} t^{r \gamma_{r}-p \gamma_{p}}-\mathcal{D}_{2} p \gamma_{p}-\mathcal{D}_{3} q \gamma_{q} t^{q \gamma_{q}-p \gamma_{p}}\right) .
$$

Denote $g(t)=t^{2-p \gamma_{p}}-\mathcal{D}_{1} \beta r \gamma_{r} t^{r \gamma_{r}-p \gamma_{p}}-\mathcal{D}_{3} q \gamma_{q} t^{q \gamma_{q}-p \gamma_{p}}$, there are

$$
\begin{gathered}
h^{\prime}(t)=t^{p \gamma_{p}-1}\left(g(t)-\mathcal{D}_{2} p \gamma_{p}\right) \\
g^{\prime}(t)=t^{1-p \gamma_{p}}\left[2-p \gamma_{p}-\mathcal{D}_{1} \beta r \gamma_{r}\left(r \gamma_{r}-p \gamma_{p}\right) t^{r \gamma_{r}-2}-\mathcal{D}_{3} q \gamma_{q}\left(q \gamma_{q}-p \gamma_{p}\right) t^{q \gamma_{q}-2}\right] .
\end{gathered}
$$


We see that $g(t)$ has a unique critical point $\bar{t}$ in $(0,+\infty)$ and

$$
\left(2-p \gamma_{p}\right) \vec{t}^{2}=\mathcal{D}_{1} \beta r \gamma_{r}\left(r \gamma_{r}-p \gamma_{p}\right) \bar{t}^{r \gamma_{r}}+\mathcal{D}_{3} q \gamma_{q}\left(q \gamma_{q}-p \gamma_{p}\right) \bar{t}^{q \gamma_{p}} .
$$

In particular, if

$$
g(\bar{t})>\mathcal{D}_{2} p \gamma_{p}, \quad h(\bar{t})>0,
$$

then $h(t)$ has exactly two critical points: one is a local minimum at a negitive level, the other on is a global maximum at positive level. Futher, there exist $0<R_{0}<R_{1}$ suct that $h\left(R_{0}\right)=h\left(R_{1}\right)=0$, and $h(t)>0$ iff $t \in\left(R_{0}, R_{1}\right)$. On the other hand, $\mathrm{A} .5$ is equivalent to

$$
\left\{\begin{array}{l}
\bar{t}^{2}>\mathcal{D}_{1} \beta r \gamma_{r} \bar{t}^{r \gamma_{r}}+\mathcal{D}_{2} p \gamma_{p} \bar{t}^{p \gamma_{p}}+\mathcal{D}_{3} q \gamma_{q} \bar{t}^{q \gamma_{q}} \\
\frac{1}{2} \bar{t}^{2}>\mathcal{D}_{1} \beta \bar{t}^{r \gamma_{r}}+\mathcal{D}_{2} \bar{t}^{p \gamma_{p}}+\mathcal{D}_{3} \bar{t}^{q \gamma_{q}}
\end{array}\right.
$$

We observe that if

$$
\bar{t}>\bar{s}:=\left(2 \mathcal{D}_{2} \min \left\{\frac{r \gamma_{r}-2}{r \gamma_{r}-p \gamma_{p}}, \frac{q \gamma_{q}-2}{q \gamma_{q}-p \gamma_{p}}\right\}\right)^{\frac{1}{2-p \gamma_{p}}},
$$

then there are

$$
\begin{aligned}
& \mathcal{D}_{1} \beta r \gamma_{r} \bar{t}^{r \gamma_{r}}+\mathcal{D}_{2} p \gamma_{p} \bar{t}^{p \gamma_{p}}+\mathcal{D}_{3} q \gamma_{q} \bar{t}^{q \gamma_{q}} \\
\leq & \max \left\{\frac{1}{r \gamma_{r}-p \gamma_{p}}, \frac{1}{q \gamma_{q}-p \gamma_{p}}\right\}\left(2-p \gamma_{p}\right) \bar{t}^{2}+\mathcal{D}_{2} q \gamma_{q} \bar{s}^{p \gamma_{p}-2} \bar{t}^{2} \\
< & \vec{t}^{2}
\end{aligned}
$$

and

$$
\mathcal{D}_{1} \beta \bar{t}^{r \gamma_{r}}+\mathcal{D}_{2} \bar{t}^{p \gamma_{p}}+\mathcal{D}_{3} \bar{t}^{q \gamma_{q}}<\frac{1}{2} \bar{t}^{2} .
$$

So we just need to guarantee $\bar{t}>\bar{s}$. Note that there exists a constant $C>0$ such that

$$
\left(2-p \gamma_{p}\right) \bar{s}^{2}>\mathcal{D}_{1} \beta r \gamma_{r}\left(r \gamma_{r}-p \gamma_{p}\right) \bar{s}^{r \gamma_{r}}+\mathcal{D}_{3} q \gamma_{q}\left(q \gamma_{q}-p \gamma_{p}\right) \bar{s}^{q \gamma_{p}}
$$

as long as

$$
\mathcal{D}_{1} \beta \mathcal{D}_{2}^{\frac{r \gamma_{r}-2}{2}-p \gamma_{p}}+\mathcal{D}_{3} \mathcal{D}_{2}^{\frac{q \gamma_{q}-2}{q \gamma_{q}-p \gamma_{p}}}<C,
$$

then $\bar{t}>\bar{s}$ because of $q \gamma_{q}, r \gamma_{q}>2$. Finally, completely analogous to Case 1, we get the constant $\alpha_{1}$ with the required properties.

Proof of Remark 3.1 The proof is similar to the one of Lemma 3.1, and we disscuss in four different cases again.

Case 1: $p \leq r<\bar{p}$. In this case, we have $p \gamma_{p} \leq r \gamma_{r}<2<q \gamma_{q}$ and

$$
l^{\prime}(t)=t^{p \gamma_{p}-1}\left(2 a t^{2-p \gamma_{p}}-b r \gamma_{r} t^{r \gamma_{r}-p \gamma_{p}}-c p \gamma_{p}-d q \gamma_{q} t^{q \gamma_{q}-p \gamma_{p}}\right) .
$$

We denote $g(t)=2 a t^{2-p \gamma_{p}}-b r \gamma_{r} t^{r \gamma_{r}-p \gamma_{p}}-d q \gamma_{q} t^{q \gamma_{q}-p \gamma_{p}}$, and hence

$$
\begin{gathered}
l^{\prime}(t)=t^{p \gamma_{p}-1}\left(g(t)-c p \gamma_{p}\right), \\
g^{\prime}(t)=t^{r \gamma_{r}-p \gamma_{p}-1}\left[\left(2-p \gamma_{p}\right) 2 a t^{2-r \gamma_{r}}-b r \gamma_{r}\left(r \gamma_{r}-p \gamma_{p}\right)-d q \gamma_{q}\left(q \gamma_{q}-p \gamma_{p}\right) t^{q \gamma_{q}-r \gamma_{r}}\right] .
\end{gathered}
$$


Now let $f(t)=\left(2-p \gamma_{p}\right) 2 a t^{2-r \gamma_{r}}-d q \gamma_{q}\left(q \gamma_{q}-p \gamma_{p}\right) t^{q \gamma_{q}-r \gamma_{r}}$, then

$$
\begin{gathered}
g^{\prime}(t)=t^{r \gamma_{r}-p \gamma_{p}-1}\left[f(t)-b r \gamma_{r}\left(r \gamma_{r}-p \gamma_{p}\right)\right] \\
f^{\prime}(t)=t^{1-r \gamma_{r}}\left[\left(2-p \gamma_{p}\right)\left(2-r \gamma_{r}\right) 2 a-d q \gamma_{q}\left(q \gamma_{q}-p \gamma_{p}\right)\left(q \gamma_{q}-r \gamma_{r}\right) t^{q \gamma_{q}-2}\right] .
\end{gathered}
$$

We see that $f(t)$ has only one critical point $\bar{t}$ in $(0,+\infty)$, which is also a maximum point, and that $f(t)$ is strictly increasing in $(0, \bar{t})$ and is strictly decreasing in $(\bar{t},+\infty)$. To obtain the monotonicity of $g(t)$, we need to compare the value of $f(\bar{t})$ and $b r \gamma_{r}\left(r \gamma_{r}-p \gamma_{p}\right)$. If $b r \gamma_{r}\left(r \gamma_{r}-p \gamma_{p}\right) \geq f(\bar{t})=\max _{t>0} f(t)$, then $g^{\prime}(t) \leq 0$ and $g(t)$ is strictly decreasing in $(0,+\infty)$. Since

$$
g(0+)= \begin{cases}0^{-}, & p<r, \\ -b r \gamma_{r}, & p=r,\end{cases}
$$

we have that $g(t)<0<c p \gamma_{p}$, and hence $l^{\prime}(t)<0$, which means that $l(t)$ has no critical points in $(0,+\infty)$. If $b r \gamma_{r}\left(r \gamma_{r}-p \gamma_{p}\right)<f(\bar{t})=\max _{t>0} f(t)$, then by $f(0)=0, f(+\infty)=-\infty$, there exist two constants $0 \leq t_{1}<\bar{t}<t_{2}$ such that $f\left(t_{1}\right)=f\left(t_{2}\right)=b r \gamma_{r}\left(r \gamma_{r}-p \gamma_{p}\right)$. So $g(t)$ is strictly decreasing for $0<t<t_{1}$ and $t>t_{2}$, and is strictly increasing for $t_{1}<t<t_{2}$. It follows form A.77 that $g(t)=c p \gamma_{p}$ has at most two solutions in $(0,+\infty)$, which implies that $l(t)$ has at most two critical points in $(0,+\infty)$.

Case 2: $r \leq p<\bar{p}$. As in Lemma 3.1, if we exchange the roles of $b t^{r \gamma_{r}}$ and $c t^{p \gamma_{p}}$, then we can conclude that $l(t)$ has at most two critical points in $(0,+\infty)$.

Case 3: $r=\bar{p}$. In this case $p \gamma_{p}<r \gamma_{r}=2<q \gamma_{q}$, and hence $l(t)$ becomes

$$
l(t)=(a-b) t^{2}-c t^{p \gamma_{p}}-d t^{q \gamma_{q}} .
$$

If $a<b$, we see that $l(t)$ is strictly decreasing and has no critical points. Now suppose $a>b$, then according to Case 1 with $p=r$, we conclude that $l(t)$ has at most two critical points in $(0,+\infty)$.

Case 4: $r>\bar{p}$. Note that in this case $p \gamma_{p}<2<r \gamma_{r}, q \gamma_{q}$. We have

$$
h^{\prime}(t)=t^{p \gamma_{p}-1}\left(2 a t^{2-p \gamma_{p}}-b r \gamma_{r} t^{r \gamma_{r}-p \gamma_{p}}-c p \gamma_{p}-d q \gamma_{q} t^{q \gamma_{q}-p \gamma_{p}}\right) .
$$

By denoting $g(t)=2 a t^{2-p \gamma_{p}}-b r \gamma_{r} t^{r \gamma_{r}-p \gamma_{p}}-d q \gamma_{q} t^{q \gamma_{q}-p \gamma_{p}}$, there are

$$
\begin{gathered}
h^{\prime}(t)=t^{p \gamma_{p}-1}\left(g(t)-c p \gamma_{p}\right), \\
g^{\prime}(t)=t^{1-p \gamma_{p}}\left[\left(2-p \gamma_{p}\right) 2 a-b r \gamma_{r}\left(r \gamma_{r}-p \gamma_{p}\right) t^{r \gamma_{r}-2}-d q \gamma_{q}\left(q \gamma_{q}-p \gamma_{p}\right) t^{q \gamma_{q}-2}\right] .
\end{gathered}
$$

Wesee that $g(t)$ has a unique critical point $\bar{t}$ in $(0,+\infty)$, which is also a maximum point, and $g(t)$ is is strictly increasing in $(0, \bar{t})$ and is strictly decreasing in $(\bar{t},+\infty)$. To obtain the monotonicity of $l(t)$, we need to compare the value of $g(\bar{t})$ and $c p \gamma_{p}$. If $c p \gamma_{p} \geq g(\bar{t})=\max _{t>0} g(t)$, then $l^{\prime}(t)<0$ and $l(t)$ has no critical points in $(0,+\infty)$. If $c p \gamma_{p}<g(\bar{t})=\max _{t>0} g(t)$, then A.77 implies that $l^{\prime}(t)=0$ has atmost two soultions in $(0,+\infty)$, that is $l(t)$ has at most two critical points in $(0,+\infty)$.

\section{B A regularity result}

We give a proof of the following facts, which is probably known, but for which we can not find a reference. 
Lemma B.1. Suppose $\Omega$ is a domain in $\mathbb{R}^{N}(N \geq 3)$ and $(u, v) \in H_{0}^{1}(\Omega) \times H_{0}^{1}(\Omega)$ is a nonnegative weak solution of

$$
\left\{\begin{array}{l}
-\Delta u=f(x, u, v), \quad \text { in } \Omega \\
-\Delta v=g(x, u, v),
\end{array}\right.
$$

where $f(x, u, v), g(x, u, v): \Omega \times \mathbb{R}^{2} \rightarrow \mathbb{R}$ are Carathéodory functions satisfying

$$
|f(x, u, v)|+|g(x, u, v)| \leq C\left(|u|+|v|+|u|^{2^{*}-1}+|v|^{2^{*}-1}\right),
$$

for some constant $C>0$. Then $(u, v)$ is a smooth solution.

Proof. We prove that $u, v \in L^{p}(\Omega)$ for any $p<\infty$ using Moser iteration, then elliptic regularity theory means that $u, v$ are smooth functions. Choose $s \geq 0$ such that $u, v \in L^{2(s+1)}(\Omega)$. We shall prove that $u \in L^{2^{*}(s+1)}(\Omega)$ so that an obvious bootstrap argument proves the assertion. Choose $L>0$ and set

$$
\psi=\min \left\{(u+v)^{s}, L\right\}, \phi=(u+v) \psi^{2}, \Omega_{L}=\left\{x \in \mathbb{R}^{N}:(u(x)+v(x))^{s} \leq L\right\} .
$$

In what follows we denote by $C$ various constants independent on $L$. We have

$$
\begin{gathered}
\nabla[(u+v) \psi]=\left(1+s \chi_{\Omega_{L}}\right) \psi \nabla(u+v), \\
\nabla \phi=\left(1+2 s \chi_{\Omega_{L}}\right) \psi^{2} \nabla(u+v),
\end{gathered}
$$

and $\phi \in H_{0}^{1}(\Omega)$. Therefore, we obtain

$$
\begin{aligned}
\int_{\Omega}|\nabla(u+v)|^{2} \psi^{2} & \leq C \int_{\Omega} \nabla(u+v) \cdot \nabla \phi=C \int_{\Omega}[f(x, u, v)+g(x, u, v)] \phi \\
& \leq C \int_{\Omega}\left(|u|+|v|+|u|^{2^{*}-1}+|v|^{2^{*}-1}\right) \phi \\
& \leq C \int_{\Omega}(|u|+|v|)^{2(s+1)}+(|u|+|v|)^{2^{*}-2}[(|u|+|v|) \psi]^{2} \\
& \leq C\left(1+\int_{\Omega} w[(|u|+|v|) \psi]^{2}\right),
\end{aligned}
$$

where $w(x)=(|u|+|v|)^{2^{*}-2} \in L^{\frac{N}{2}}(\Omega)$. Then we obtain

$$
\begin{aligned}
\int_{\Omega}|\nabla[(u+v) \psi]|^{2} & \leq C \int_{\Omega}|\nabla(u+v)|^{2} \psi^{2} \leq C\left(1+\int_{\Omega} w[(|u|+|v|) \psi]^{2}\right) \\
& \left.\leq C\left(1+K \int_{|w| \leq K}(|u|+|v|)^{2(s+1)}+\int_{|w|>K} w[(|u|+|v|) \psi]^{2}\right)\right) \\
& \leq C\left(1+K+\left(\int_{|w|>K} w \frac{N}{2}\right)^{\frac{2}{N}}\left(\int_{\Omega}[(u+v) \psi]^{2^{*}}\right)^{\frac{2}{2^{*}}}\right) \\
& \leq C(1+K)+\varepsilon_{K} \int_{\Omega}|\nabla[(u+v) \psi]|^{2}
\end{aligned}
$$

where $\varepsilon_{K} \rightarrow 0$ as $K \rightarrow+\infty$. Choosing $K$ such that $\varepsilon_{K}<\frac{1}{2}$ we arrive at

$$
\int_{\Omega_{L}}\left|\nabla(u+v)^{s+1}\right|^{2}=\int_{\Omega_{L}}|\nabla[(u+v) \psi]|^{2} \leq C .
$$

Letting $L \rightarrow+\infty$, we get $u^{s+1}, v^{s+1} \in H^{1}(\Omega)$, hence $u \in L^{2^{*}(s+1)}(\Omega)$. 
Corollary B.1. Any nonnegative solution of 1.2 is smooth solution.

Proof. In this case, $\Omega=\mathbb{R}^{N}$ and

$$
\begin{aligned}
& f(x, u, v)=-\lambda_{1} u+\mu_{1}|u|^{p-2} u+\beta r_{1}|u|^{r_{1}-2}|v|^{r_{2}} u \\
& g(x, u, v)=-\lambda_{2} v+\mu_{2}|v|^{q-2} v+\beta r_{2}|u|^{r_{1}}|v|^{r_{2}-2} v,
\end{aligned}
$$

then by Young inequality we have

$$
\begin{aligned}
|f(x, u, v)|+|g(x, u, v)| & \leq C\left(|u|+|v|+|u|^{p-1}+|v|^{q-1}+|u|^{r-1}+|v|^{r-1}\right) \\
& \leq C\left(|u|+|v|+|u|^{2^{*}-1}+|v|^{2^{*}-1}\right) .
\end{aligned}
$$

Then from Lemma B.1, we obtain any nonnegative solution of (1.2) is smooth.

\section{Acknowledgements}

The authors thank Nicola Soave for valuable comments for preparing the current manuscript: he pointed out a gap in Lemma 3.5 and gave some comments for the Theorem 1.2 .

\section{References}

[1] S. K. Adhikari. Superfluid Fermi-Fermi mixture:phase diagram, stability, and solition formation. Phys. Rev., A 76(2007), 053609.

[2] V. S. Bagnato, D. J. Frantzeskakis, P. G. Kevrekidis B. A. Malomed and D. Mihalache. Bose-Einstein condensation: twenty years after. Roman. Rep. Phys., 67(2015), 5-50.

[3] T. Bartsch and N. Soave. A natural constraint approach to normalized solutions of nonlinear Schrödinger equations and systems. J. Funct. Anal., 272(2017), no.12, 4998-5037.

[4] T. Bartsch and L. Jeanjean. Normalized solutions for nonlinear Schrödinger systems. Proc. Roy. Soc. Edinburgh Sect. A, 148(2018), no.2, 225-242.

[5] T. Bartsch, L. Jeanjean and N. Soave. Normalized solutions for a system of coupled cubic Schrödinger equations on $\mathbb{R}^{3}$. J. Math. Pures Appl., (9)106(2016), no.4, 583-614.

[6] T. Bartsch and N. Soave. Multiple normalized solutions for a competing system of Schrödinger equations. Calc. Var. Partial Differential Equations, 58(2019), no.1, Art.22, 24 pp.

[7] Z. Chen and W. Zou. Positive least energy solutions and phase separation for coupled Schrödinger equations with critical exponent: higher dimensional case. Calc. Var. Partial Differential Equations, 52(2015), no.1-2, 423-467.

[8] B. D. Esry, C. H. Greene, J. P. Burke Jr and J. L. Bohn. Hartree-Fock theory for double condensates. Phys. Rev. Lett., 78(1997), 3594.

[9] N. Ghoussoub. Duality and perturbation methods in critical point theory. 1993.

[10] B. Gidas, W. Ni and L. Nirenberg. Symmetry and related properties via the maximum principle. Comm. Math. Phys., 68(1979), no.3, 209-243. 
[11] T. Gou and L. Jeanjean. Existence and orbital stability of standing waves for nonlinear Schrödinger systems. Nonlinear Anal., 144(2016), 10-22.

[12] T. Gou and L. Jeanjean. Multiple positive normalized solutions for nonlinear Schrödinger systems. Nonlinearity, 31(2018), no.5, 2319-2345.

[13] N. Ikoma. Compactness of minimizing sequences in nonlinear Schrödinger systems under multiconstraint conditions. Adv. Nonlinear Stud., 14(2014), no.1, 115-136.

[14] N. Ikoma, K. Tanaka. A note on deformation argument for L2 normalized solutions of nonlinear Schrödinger equations and systems. Adv. Differential Equations, 24(2019), 609-646.

[15] L. Jeanjean. Existence of solutions with prescribed norm for semilinear elliptic equations. Nonlinear Anal., 28(1997), no.10, 1633-1659.

[16] M. K. Kwong. Uniqueness of positive solutions of $\Delta u-u+u^{p}=0$ in $\mathbb{R}^{n}$. Arch. Rational Mech. Anal., 105(1989), no.3, 243-266.

[17] Y. Li and W. Ni. Radial symmetry of positive solutions of nonlinear elliptic equations in $\mathbb{R}^{N}$. Comm. Partial Differential Equations, 18(1993), no.5-6, 1043-1054.

[18] E. H. Lieb and M. Loss. Analysis. Second edition. 2001.

[19] B. Malomed. Multi-Component Bose-Einstein Condensates: Theory. 2008.

[20] P. Quittner and P. Souplet. Superlinear parabolic problems. Blow-up, global existence and steady states. 2007.

[21] N. Soave. Normalized ground states for the NLS equation with combined nonlinearities. J. Differential Equations, 269(2020), no.9, 6941-6987.

[22] N. Soave. Normalized ground states for the NLS equation with combined nonlinearities: the Sobolev critical case. J. Funct. Anal., 279(2019), no.6. 